\title{
Characteristics and Mechanism Analysis of Aerodynamic Noise Sources for High-Speed Train in Tunnel
}

\author{
Xiao-Ming Tan, Hui-fang Liu, Zhi-Gang Yang $(\mathbb{D}$, Jie Zhang, Zhong-gang Wang, \\ and Yu-wei Wu
}

Key Laboratory of Traffic Safety on Track, Ministry of Education, Central South University, Changsha,
Hunan Province 410075, China

Correspondence should be addressed to Zhi-Gang Yang; sound_wdq@csu.edu.cn

Received 21 May 2018; Revised 2 September 2018; Accepted 25 September 2018; Published 9 December 2018

Academic Editor: Mohammad Hassan Khooban

Copyright ( 2018 Xiao-Ming Tan et al. This is an open access article distributed under the Creative Commons Attribution License, which permits unrestricted use, distribution, and reproduction in any medium, provided the original work is properly cited.

\begin{abstract}
We aim to study the characteristics and mechanism of the aerodynamic noise sources for a high-speed train in a tunnel at the speeds of $50 \mathrm{~m} / \mathrm{s}, 70 \mathrm{~m} / \mathrm{s}, 83 \mathrm{~m} / \mathrm{s}$, and $97 \mathrm{~m} / \mathrm{s}$ by means of the numerical wind tunnel model and the nonreflective boundary condition. First, the large eddy simulation model was used to simulate the fluctuating flow field around a 1/8 scale model of a high-speed train that consists of three connected vehicles with bogies in the tunnel. Next, the spectral characteristics of the aerodynamic noise source for the high-speed train were obtained by performing a Fourier transform on the fluctuating pressure. Finally, the mechanism of the aerodynamic noise was studied using the sound theory of cavity flow and the flow field structure. The results show that the spectrum pattern of the sound source energy presented broadband and multipeak characteristics for the highspeed train. The dominant distribution frequency range is from $100 \mathrm{~Hz}$ to $4 \mathrm{kHz}$ for the high-speed train, accounting for approximately $95.1 \%$ of the total sound source energy. The peak frequencies are $400 \mathrm{~Hz}$ and $800 \mathrm{~Hz}$. The sound source energy at $400 \mathrm{~Hz}$ and $800 \mathrm{~Hz}$ is primarily from the bogie cavities. The spectrum pattern of the sound source energy has frequency similarity for the bottom structure of the streamlined part of the head vehicle. The induced mode of the sound source energy is probably the dynamic oscillation mode of the cavity and the resonant oscillation mode of the cavity for the under-car structure at $400 \mathrm{~Hz}$ and $800 \mathrm{~Hz}$, respectively. The numerical computation model was checked by the wind tunnel test results.
\end{abstract}

\section{Introduction}

High-speed trains have become the preferred vehicle for middle- and long-distance travel. They are critical in social economic development and cultural exchange and have attracted wide attention worldwide [1, 2]. However, with the further increase in their operating speed $(>300 \mathrm{~km} / \mathrm{h})$, the problem of environmental pollution of high-speed trains due to aerodynamic noise has become an urgent problem that needs to be solved by producers and operators. The comprehensive and accurate understanding of aerodynamic noise characteristics and its generation mechanism is the basis of aerodynamic noise control of high-speed trains. After more than 20 years of wind tunnel tests, field running tests, and numerical simulations, researchers have attained a comprehensive and accurate understanding of the aerodynamic noise characteristics and the generation mechanism of high-speed trains.

Before the previous century, it was widely believed that the operating speed of $300 \mathrm{~km} / \mathrm{h}$ was the acoustic transition speed $[3,4]$ of high-speed trains. However, with the gradual application of noise reduction techniques such as the streamlined head shape and the smooth car body in high-speed train design, the acoustic conversion speed of high-speed trains is increased to $350 \mathrm{~km} / \mathrm{h}$ [5-7]. The speed of acoustic conversion is defined as the operating speed of high-speed trains when the contributions of the aerodynamic noise and rolling noise are the same.

The dominant noise sources of different high-speed trains are not identical, but the bogie area and the pantograph area are generally recognized as the dominant sources of exterior noise. In the field running test, Mellet et al. [8] 
used the acoustic imaging technology to identify the dominant noise sources for the ICE high-speed train, which were the streamline part of head/tail car, the bogie area, the pantograph area, and the ventilation area, but did not include the intercoach windshield area; He et al. [9] also used the similar method to identify the dominant noise sources for a type of Chinese high-speed train, which were the bogie area, the pantograph area, and the intercoach windshield area; Deng et al. [10] also utilized the similar method to identify the dominant noise sources for another type of Chinese high-speed train, which included the streamline part of the head, the intercoach windshield area, the bogie area, and the pantograph area.

The spectrum characteristics and propagation characteristics of the aerodynamic noises in the bogie area, the pantograph area, and the intercoach windshield area have gradually become the focus of research in recent years. In 2000, Frémion et al. [11] used coherent output power technology to analyze the spectral characteristics of the aerodynamic noises in the intercoach windshield area and the bogie area of the full-size French TGV train. They found that the radiated noise in the intercoach windshield area was primarily tonal and distributed in the low-frequency range, but had little influence on the total aerodynamic noise. The bogie area had a variety of uncorrelated sound sources. The external radiation was insignificant from the sound source at the bottom of the bogie, but was relatively obvious from the upstream and the downstream of the wheel arches. The dominant frequency distribution range was 500$1000 \mathrm{~Hz}$ for the noises radiated from the upstream and downstream of the wheel arches. The spectrum of the sound source located downstream of the wheel arches had multipeak frequencies near $600 \mathrm{~Hz}$. In 2012, Lauterbach et al. [12] studied the Reynolds number effect of aerodynamic noise on a $1 / 25$ scale model of a high-speed train in the aeroacoustic wind tunnel of the German Aerospace Center in Brunswick and in the cryogenic wind tunnel of the DNW (German-Dutch wind tunnels) in Cologne. They found that the aerodynamic noise in the bogie area at 1:25 1:25 was primarily distributed below $5000 \mathrm{~Hz}$ and can be described by the cavity excitation mode. The aerodynamic noise in the pantograph area was primarily distributed above $5000 \mathrm{~Hz}$ and has the Strouhal number similarity. In 2015, Lee et al. [13] numerically analyzed the contribution of the pantograph's various components to the aerodynamic noise energy at $400 \mathrm{~km} / \mathrm{h}$ in the closed state and indicated that the aerodynamic noise at $(60-400 \mathrm{~Hz})$, $(600-800 \mathrm{~Hz}),(1 \mathrm{kHz}-2 \mathrm{kHz})$, and $(2 \mathrm{kHz}-5 \mathrm{kHz})$ was primarily derived from the bottom frame area, the panhead area, the knuckle area between the upper and lower arms, and the whole pantograph, respectively. In 2017, Iglesias et al. [14] discovered that the trailing edge of the bogie cavity contributed highly to the noise radiated from the bogie cavity using sound source imaging technology in the anechoic wind tunnel test of the Japan Railway Technology Research Institute. Their results are consistent with the conclusion of Yamazaki and Ido [15].

Sound analogy theory is the dominant theoretical basis for the current research on the aerodynamic noise generation mechanism of high-speed trains. Dipole noise sources are generally considered to be the dominant noise source [16] for high-speed trains and can be described by the fluctuating pressure [17] on the train surface. The fluctuating pressure on the train surface is closely related to the structure of the flow field and the induced mechanism of the flow field structure in the different areas for the high-speed train. For the head car part, the combination of the effect of airflow, the airflow separation and reattachment of streamlined shoulder, the airflow blending in the cowcatcher area, and vortex shedding creates a unique flow field structure [18] in the area. For the middle train, the hairpin vortex in the turbulent boundary layer dominates the momentum transmission on the train surface, which is an important mechanism for the generation and dissipation of turbulence in the area [19]. For the tail car part, the streamlined shoulder flow has a large-scale separation and reattachment, and the vortex structures produced by the tail car and its upstream components are highly mixed in the recirculation zone, forming the flow field characteristics of the wake region with high turbulence intensity, large turbulent kinetic energy, and a pair of oppositely directed large-scale drag vortex structures [20-22]. For the intercoach windshield area, the cavity vocalization is its dominant vocal mechanism [23, 24]. For the pantograph area, the airflow separation and the vortex shedding from the rod and their interactions are the dominant reasons for the flow field structure in this area [17, 25, 26]. For the bogie area, the flow pattern of the cavity and the bluff body flow pattern such as the wheelset and axle are combined in the narrow space of the bogie cavity, which is the dominant inducing mechanism of the complex flow field structure in this area $[27,28]$.

It is noteworthy that the research results above are all directed to high-speed trains on the open track and lack the detailed studies on the aerodynamic noise characteristics and the mechanism of the high-speed trains in tunnels. The flow field structure-induced mechanism on an open track and the wave interference mechanism in a tunnel cause the flow structure of the high-speed train in the tunnel to be complicated. The sliding grid method is the dominant research method for the current aerodynamics problems of high-speed trains crossing tunnels [29, 30]. However, the most significant drawback of this method is the interpolation error of the data exchange surface, resulting in inconsistent flow fluxes on both sides, and thus, the flow spectrum jumps on the data exchange surface. This jump has little influence on the aerodynamic issues such as drag, lift, and torque. However, it has a significant influence on the calculation of the fluctuating quantity of the flow field. Therefore, the sliding grid method is generally not recommended in aeroacoustic calculations [31]. Currently, numerical models of high-speed train's aerodynamic noise on an open track tends to be mature and can accurately predict the aerodynamic noise of high-speed trains. This paper attempts to introduce a nonreflective boundary condition based on the numerical model of aerodynamic noise calculation for high-speed trains on an open track to study the flow field structure, the aerodynamic noise source 
intensity distribution characteristics/spectral characteristics, and their mutual relations in an infinite tunnel. The Reynolds numbers based on vehicle height and incoming velocity are $1.69 \times 10^{6}, 2.36 \times 10^{6}, 2.80 \times 10^{6}, 3.28 \times 10^{6}$, respectively. This paper is structured as follows: Section 2 introduces the turbulence model, acoustic reflection-free boundary, and acoustic equations used herein. Section 3 establishes and verifies the numerical model of the aerodynamic noise of a high-speed train in a tunnel. Section 4 discusses the flow field structure of high-speed trains in tunnels. Section 5 analyzes the distribution/spectrum characteristics and velocity dependence law of the noise source on the high-speed train in tunnels. Section 6 analyzes the aerodynamic noise mechanism of high-speed trains in tunnels using the flow field structure and the cavity flowsound theory. Section 7 presents the conclusion.

\section{Mathematical Physical Model}

2.1. Large Eddy Simulation (LES) Model. Although the running Mach number of high-speed trains herein is less than 0.3 , the influence of the change in air density on the flow field around the train in the tunnel cannot be ignored. Therefore, the three-dimensional, unsteady, compressible, and viscous Navier-Stokes (N-S) equations are used herein. The LES model can well capture the vortices and flow field fluctuating information and thus is suitable for refined flow field simulations. LES is a special filtering method used to filter the compressible N-S equations to obtain the compressible numerical simulation control equations describing the large-scale vortex motion, while the subgrid scale model is used to solve the small-scale vortices to avoid the direct simulation of the full-scale eddy motion of the flow field. The Favre filtering method is a relatively simple and practical filtering method, which uses the common physical space filtering for density and pressure and density-weighted filtering for speed, temperature, and internal energy. The compressible numerical simulation control equation filtered by the Favre filtering [32] method is as follows:

$$
\begin{aligned}
\frac{\partial \bar{\rho}}{\partial t}+\frac{\partial \bar{\rho}_{i}}{\partial x_{i}} & =0, \\
\frac{\partial \bar{\rho} \widehat{u}_{i}}{\partial t}+\frac{\partial \bar{\rho} \widehat{u}_{i} \widehat{u}_{j}}{\partial x_{j}} & =-\frac{\partial \bar{p}}{\partial x_{i}}+\frac{\partial \widehat{\sigma}_{i j}}{\partial x_{j}}+\frac{\partial \widehat{\tau}_{i j}}{\partial x_{j}}, \\
\left.\frac{\partial(\bar{\rho} \widehat{e}+}{\rho} \widehat{u}_{i} \widehat{u}_{i} / 2\right) & +\frac{\partial\left(\bar{\rho} \widehat{e}+\bar{\rho}_{i} \widehat{u}_{i} / 2+\bar{p}\right) \widehat{u}_{j}}{\partial x_{j}} \\
& =\frac{\partial \widehat{\sigma}_{i j} \widehat{u}_{i}}{\partial x_{j}}+\frac{\partial}{\partial x_{j}}\left(\widehat{\lambda} \frac{\partial \widehat{T}}{\partial x_{j}}\right)+\widehat{A},
\end{aligned}
$$

where “-" represents the physical space filtering, “ $\wedge$ " represents the density-weighted filtering, $i$ and $j$ are the minor scales, $\rho$ is the air density, $p$ is the pressure, $u$ is the flow speed, $\mu$ is the dynamic viscosity coefficient, $\lambda$ is the thermal conductivity, $e$ is the internal energy, and
$T$ is the temperature. The equations for $\widehat{\sigma}_{i j}, \widehat{\tau}_{i j}$, and $\widehat{A}$ are shown as follows:

$$
\begin{aligned}
\widehat{\sigma}_{i j}= & \mu\left(\frac{\partial \widehat{u}_{i}}{\partial x_{j}}+\frac{\partial \widehat{u}_{j}}{\partial x_{i}}\right), \\
\widehat{\tau}_{i j}= & \bar{\rho}\left(\widehat{u}_{i} \widehat{u}_{j}-u_{i} u_{j}\right), \\
\widehat{A}= & \widehat{u}_{i} \frac{\partial \bar{\rho}\left(\widehat{u}_{i} \widehat{u}_{j}-u_{i} u_{j}\right)}{\partial x_{j}}-\frac{\partial\left(\overline{e u_{j}}-\bar{e}_{j}\right)}{\partial x_{j}} \\
& -\left(\overline{\left.\partial \frac{\partial u_{j}}{\partial x_{j}}-\bar{p} \frac{\partial \widehat{u}_{j}}{\partial x_{j}}\right)}+\left(\overline{\sigma_{i j} \frac{\partial u_{i}}{\partial x_{j}}-\bar{\sigma}_{i j}} \frac{\partial \widehat{u}_{i}}{\partial x_{j}}\right)\right. \\
& +\frac{\partial\left(\bar{\sigma}_{i j} \widehat{u}_{i}-\widehat{\sigma}_{i j} \widehat{u}_{i}\right)}{\partial x_{j}}+\frac{\partial}{\partial x_{j}}\left(\bar{\lambda} \frac{\partial \bar{T}}{\partial x_{j}}-\widehat{\lambda} \frac{\partial \widehat{T}}{\partial x_{j}}\right) .
\end{aligned}
$$

The density-weighted filtered state equation is

$$
\bar{p}=\bar{\rho} R \widehat{T}
$$

where $R$ is the gas constant.

To close the system of equations, the subgrid scale (SGS) model proposed by Smagorinsky is introduced to the subgrid stress $[33,34]$.

2.2. Nonreflecting Boundary Condition. The nonreflective boundary condition can effectively solve the problem of the pseudoreflected waves caused by the artificial truncated computational domain, thereby enabling the simulation of the flow field around the high-speed train in an infinitely long tunnel. By reconstructing the Euler equations based on the wave characteristics, a set of equations acoustically describing nonreflective boundary conditions can be obtained [35-37] as follows:

$$
\begin{array}{r}
\frac{\partial \rho}{\partial t}+d_{1}+\frac{\partial \rho u_{2}}{\partial x_{2}}+\frac{\partial \rho u_{3}}{\partial x_{3}}=0, \\
\frac{\partial \rho u_{1}}{\partial t}+u_{1} d_{1}+\rho d_{3}+\frac{\partial\left(\rho u_{1} u_{2}\right)}{\partial x_{2}}+\frac{\partial\left(\rho u_{1} u_{3}\right)}{\partial x_{3}}=0, \\
\frac{\partial \rho u_{2}}{\partial t}+u_{2} d_{1}+\rho d_{4}+\frac{\partial\left(\rho u_{2} u_{2}\right)}{\partial x_{2}}+\frac{\partial\left(\rho u_{2} u_{3}\right)}{\partial x_{3}}+\frac{\partial p}{x_{2}}=0, \\
\frac{\partial \rho u_{3}}{\partial t}+u_{3} d_{1}+\rho d_{5}+\frac{\partial\left(\rho u_{3} u_{2}\right)}{\partial x_{2}}+\frac{\partial\left(\rho u_{3} u_{3}\right)}{\partial x_{3}}+\frac{\partial p}{x_{3}}=0, \\
\frac{\partial \rho e}{\partial t}+\frac{1}{2}\left(u_{1}^{2}+u_{2}^{2}+u_{3}^{2}\right) d_{1}+\frac{d_{2}}{\gamma-1}+\rho u_{1} d_{3}+\rho u_{2} d_{4} \\
+\rho u_{3} d_{5}+\frac{\partial\left[(\rho e+p) u_{2}\right]}{\partial x_{2}}+\frac{\partial\left[(\rho e+p) u_{3}\right]}{\partial x_{3}}=0 .
\end{array}
$$

The coordinate system is a local Cartesian coordinate system based on a nonreflective boundary, where the $x$-axis is perpendicular to the boundary and points outward; $c$ 
is the speed of sound. The formula for each coefficient is as follows:

$$
\begin{aligned}
& d_{1}=\frac{1}{c^{2}}\left[L_{2}+\frac{1}{2}\left(L_{5}+L_{1}\right)\right], \\
& d_{2}=\frac{1}{2}\left(L_{5}+L_{1}\right), \\
& d_{3}=\frac{1}{2 \rho c}\left(L_{5}-L_{1}\right), \\
& d_{4}=L_{3}, \\
& d_{5}=L_{4}, \\
& L_{1}=\left(u_{1}-c\right)\left(\frac{\partial p}{\partial x_{1}}-\rho c \frac{\partial u_{1}}{\partial x_{1}}\right), \\
& L_{2}=u_{1}\left(c^{2}-1\right) \frac{\partial p}{\partial x_{1}}, \\
& L_{3}=u_{1} \frac{\partial u_{2}}{\partial x_{1}}, \\
& L_{4}=u_{1} \frac{\partial u_{3}}{\partial x_{1}}, \\
& L_{5}=\left(u_{1}+c\right)\left(\frac{\partial p}{\partial x_{1}}+\rho c \frac{\partial u_{1}}{\partial x_{1}}\right) .
\end{aligned}
$$

2.3. Aeroacoustic Equation. The Lighthill's sound analogy theory $[16,38]$ divides the sound sources into three types: monopole, dipole, and quadrupole. In practical applications, the dominant types of sound sources are selected for integration, which facilitates engineering applications. Herein, the train is a stationary rigid solid wall, and the velocity of airflow passing through its surface is subsonic. Therefore, the Curle acoustics integral formula [39] can be used to study the aerodynamic sound from the train. The Curle acoustics integral formula is as follows:

$$
p_{\text {sound }}(\mathbf{x}, t)=\frac{1}{4 \pi c_{0}} \int_{s} \frac{\cos (\theta)}{r} \frac{\partial}{\partial t} p(\mathbf{y}, \tau) d S(\mathbf{y})
$$

where $p_{\text {sound }}$ is the sound pressure, $\mathbf{x}$ and $\mathbf{y}$ are, respectively, the spatial location vectors of the far-field receiver and the sound source, $c_{0}$ is the sound speed in the far field, $\theta$ is the angle between the surface normal vector of the sound source and the vector from the sound source to the far-field receiver, $r$ is the distance from the sound source to the far-field receiver, $\tau$ is the retarded time, $S$ is the surface area of the sound source, and $p$ is the fluctuating pressure on the train surface.

Using (6), the acoustic energy density of the far-field aerodynamic noise $I$ can be approximated as follows:

$$
I \propto \overline{p_{\text {sound }}{ }^{2}(\mathbf{x}, t)}=\frac{1}{16 \pi^{2} c_{0}^{2}} \overline{\left(\int_{s} \frac{\cos (\theta)}{r} \frac{\partial}{\partial t} p(\mathbf{y}, \tau) d S(\mathbf{y})\right)^{2}}
$$

where the overline represents the average values of the physical quantities in the time domain.

In the far field, the size of the sound source is negligible. It can be regarded as a point sound source. Subsequently, $r$ is approximately equal to $R$. Again, the flow rate is a low Mach number such that the effect of delay time can be ignored. Thus, the total acoustic power $W_{\text {rad }}$ radiated from the sound source can be expressed as follows:

$$
\begin{aligned}
W_{\text {rad }} & \propto \frac{4 \pi R^{2}}{3} \overline{p_{\text {sound }}^{2}}(\mathbf{x}, t) \\
& =\frac{4 \pi R^{2}}{3} \frac{1}{16 \pi^{2} c_{0}^{2}} \overline{\left(\int_{s} \frac{\cos (\theta)}{r} \frac{\partial}{\partial t} p(\mathbf{y}, \tau) d S(\mathbf{y})\right)^{2}} \\
& =\frac{1}{12 \pi c_{0}^{2}} \overline{\left(\int_{s} \cos (\theta) \frac{\partial}{\partial t} p(\mathbf{y}) d S(\mathbf{y})\right)^{2}},
\end{aligned}
$$

where $R$ is the sphere radius of the acoustic energy integral surface.

In $(8),(\partial / \partial t) p(\mathbf{y})$ represents the intensity of the sound source, and $\cos (\theta)(\partial / \partial t) p(\mathbf{y})$ represents the projection of the intensity of the sound source in the direction from the sound source to the receiver. Therefore, from the aspect of engineering application, we may define the acoustic power of the sound source as follows:

$$
W_{\text {source }} \propto \overline{\left(\int_{s} \frac{\partial}{\partial t} p(\mathbf{y}) d S(\mathbf{y})\right)^{2}}=\overline{\left(\frac{\partial}{\partial t} \int_{s} p(\mathbf{y}) d S(\mathbf{y})\right)^{2}}
$$

We define

$$
F=\int_{s} p(\mathbf{y}) d S(\mathbf{y})
$$

Subsequently,

$$
W_{\text {source }} \propto \overline{{F^{\prime 2}}^{2}}
$$

where the right superscript ' represents the time derivative, $W_{\text {source }}$ is the acoustic power of the sound source, and $F$ is the fluctuating force.

It is noteworthy that the calculation formula of the acoustic power of the sound source does not consider the sound source radiation characteristics, and that this paper studies only dipole noise sources. Herein, (11) will be applied to calculate the percentage of the sound source energy from each component of the high-speed train in the total sound source energy.

\section{Numerical Computation Model}

3.1. Model Preparation. The geometric model used herein is a high-speed train with three cars, with bogies and without pantographs at a scale of $1: 8$. The full-size model has a length of $79.6 \mathrm{~m}$, a height of $4.08 \mathrm{~m}$, and a width of $3.36 \mathrm{~m}$. The names of the various parts of the model are as follows: the bogies from the upstream to the downstream are named the 


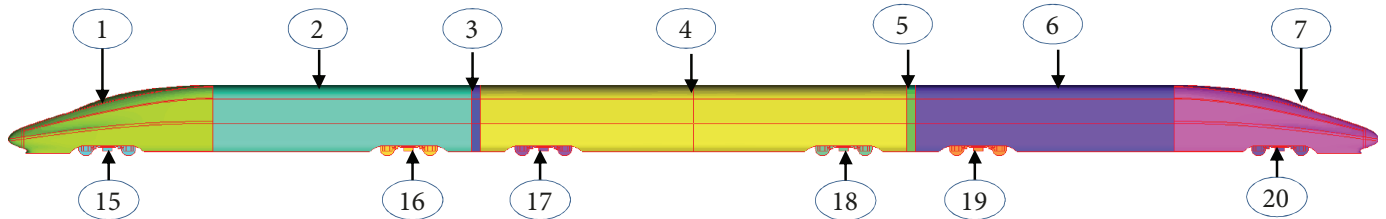

(a)

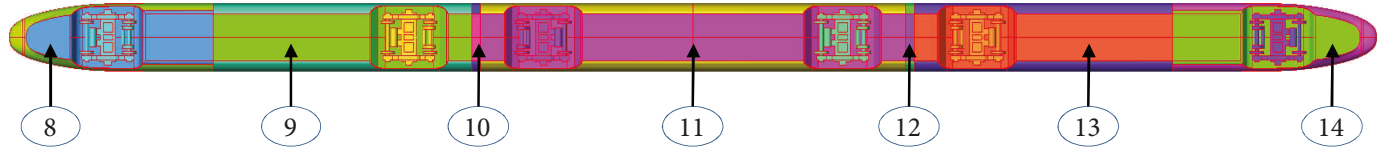

(b)

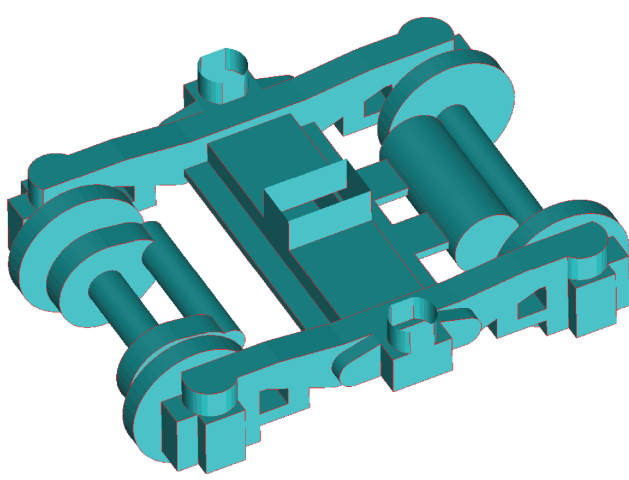

(c)

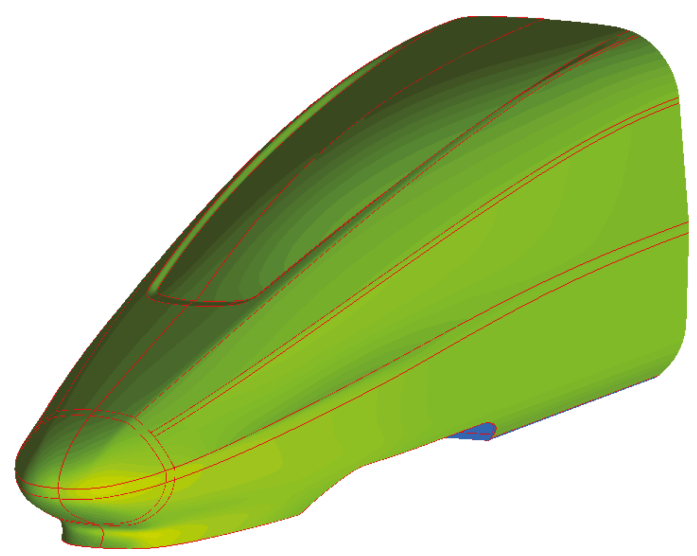

(d)

FIgURE 1: Geometric structure of the high-speed train: (a) front view, (b) bottom view, (c) bogie, and (d) the streamlined part. The list of components in the figure is as follows. 1: the upper part of the head car's streamlined part; 2: the upper part of the head car's carriage; 3: the upper part of the intercoach windshield 1; 4: the upper part of the mid-car; 5: the upper part of the intercoach windshield 2; 6: the upper part of the tail car's carriage; 7: the upper part of the tail car's streamlined part; 8: the bottom of the head car's streamlined part; 9: the bottom of the head car's carriage; 10: the bottom of the intercoach windshield 1; 11: the bottom of the mid-car; 12: the bottom of the intercoach windshield 2; 13: the bottom of the tail car's carriage; 14: the upper part of the head car's streamlined part; 15: bogie 1; 16: bogie 2; 17: bogie 3; 18: bogie 4; 19: bogie 5; and 20: bogie 6 .

first bogie to the sixth bogie, sequentially; the three cars from the upstream to the downstream were named the head car, the mid-car, and the tail car, sequentially. The streamlined parts of the head car and the tail car are named independently. The bottom of each carriage is independently named. A schematic view of the train is shown in Figure 1.

As shown in Figure 2, the cross section of the full-sized computational domain is fan shaped, with a radius of $6.87 \mathrm{~m}$, an area of $100 \mathrm{~m}^{2}$, and a blockage ratio of 0.136 . In the $x$-direction, the head car is $400 \mathrm{~m}$ away from the inlet and the tail car is $800 \mathrm{~m}$ away from the outlet. In the $y$-direction, the whole fluid field is symmetrical approximately the longitudinal symmetry of the train. The lowest end of the wheelset is approximately $0.2 \mathrm{~m}$ above the ground. The inlet of the tunnel is set as the pressure inlet and nonreflecting boundary condition, and the outlet of the tunnel is set as the pressure outlet and nonreflecting boundary condition. The high-speed train is set as a noslip wall boundary condition. The tunnel is set as a slipping wall with a speed equal to the train running speed.

In this study, the topological optimization, the multiple density box, the boundary layer mesh, and the extruding

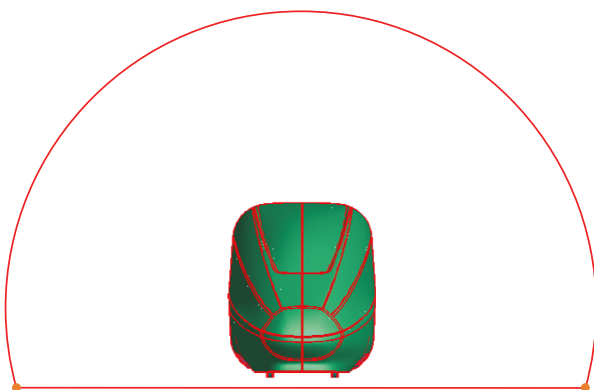

Figure 2: Computational domain.

mesh of the commercial meshing software ICEM were used to perform a refined tetrahedron/triangular prism meshing, as shown in Figure 3. The triangular mesh sizes of the head/tail car's streamlined part, the head/tail car's carriage, the mid-car, six bogies, the tunnel's inlet/outlet, and the tunnel wall were $40 \mathrm{~mm}, 50 \mathrm{~mm}, 60 \mathrm{~mm}, 40 \mathrm{~mm}, 500 \mathrm{~mm}$, and $500 \mathrm{~mm}$, respectively. The mesh was properly refined for larger curved surfaces. The minimum/largest mesh sizes of the whole domain were $10 \mathrm{~mm}$ and $500 \mathrm{~mm}$, respectively. 


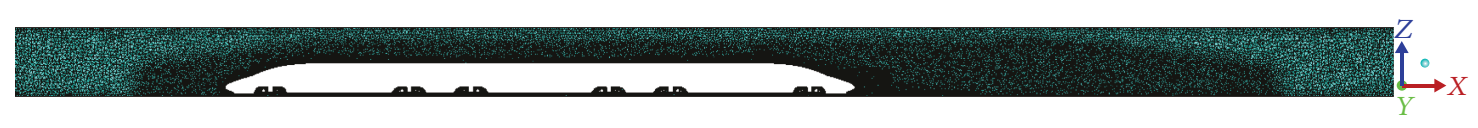

(a)

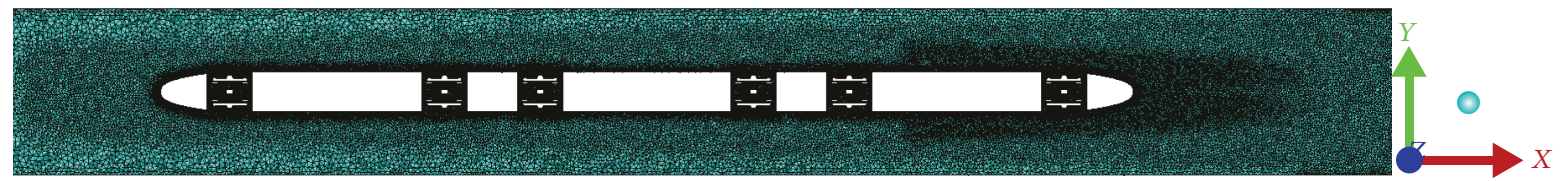

(b)

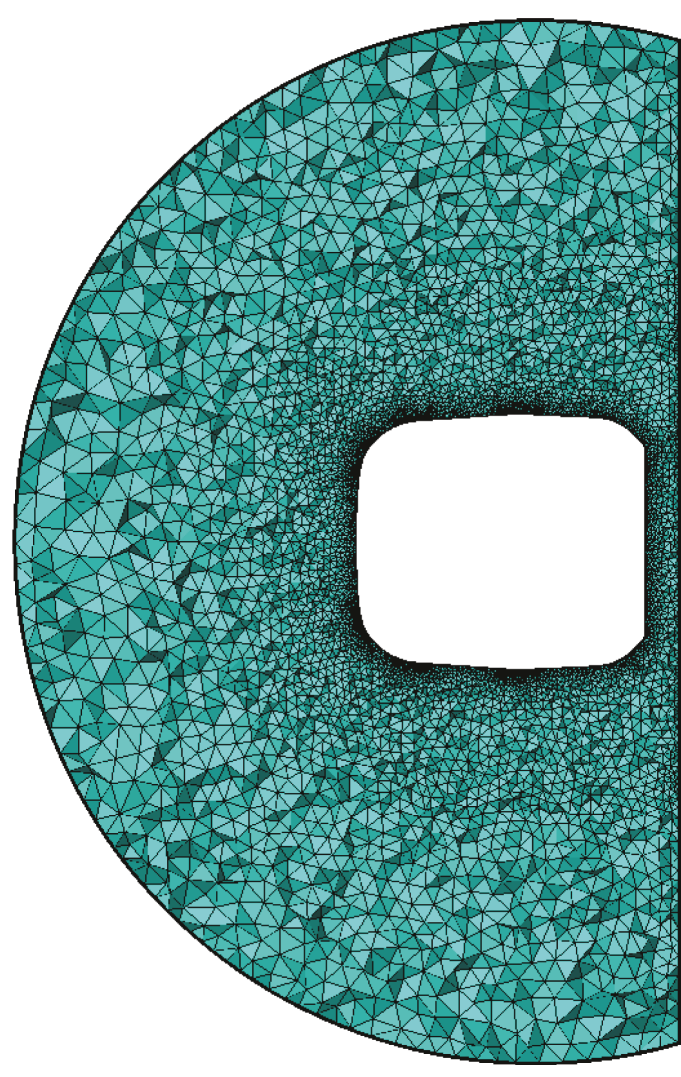

(c)

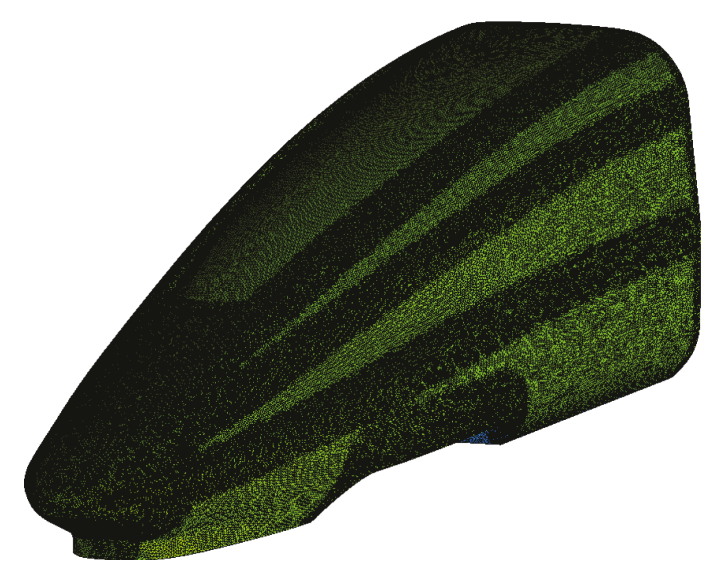

(d)

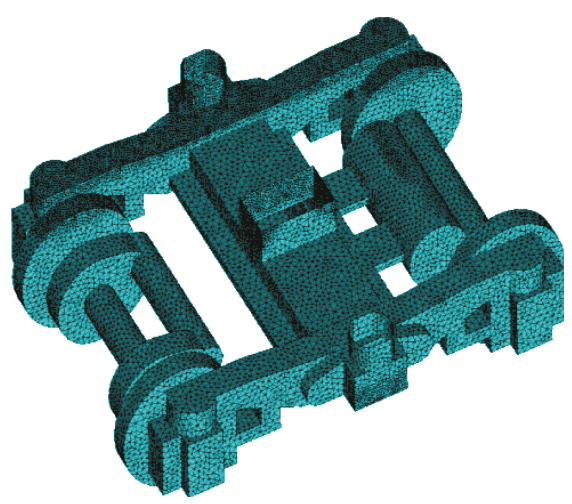

(e)

FIGURE 3: Mesh distribution: (a) longitudinal plane, (b) $x-y$ plane with a height of $0.1 \mathrm{~m}$ from the ground, (c) cross section of the streamlined shoulder, (d) streamlined part, and (e) bogie.

Further, 25 layers of boundary layer triangular prism mesh were added to the train surface. The thickness of the first layer was $0.1 \mathrm{~mm}\left(y^{+}<1\right)$, and the grid growth rate was 1.03. The number of triangular prism layers on the tunnel wall was eight. The thickness of the first layer was $10 \mathrm{~mm}$, and the grid growth rate was 1.1. Considering that the region where the flow field around the train changes rapidly gradually widens downstream, the area five times the train height from the train tail is the area where the wake vortex is generated and developed; the streamlined two-sided area of the tail car shows an obvious mixing effect, and the air separation occurs at the shoulder of the tail streamlined part. Seven density boxes were set up: one density box was a thin block that enveloped the shoulder of the tail streamlined part and adopted the $50 \mathrm{~mm}$ mesh size; two density boxes were wedges close to the sides of the tail streamlined part, with a mesh size of $50 \mathrm{~mm}$; one density box was a square that enclosed the nose and the cowcatcher of the tail car. The length of this square was approximately 0.5 times the height of the train, and this region was divided into a tetrahedral mesh with a grid scale of $50 \mathrm{~mm}$. The other three nested density boxes were composed of three truncated pyramids. The three front, middle, and rear prisms, respectively, enveloped the streamlined part of the head train, the head/tail/middle carriages, and the tail streamlined part. The width of the downstream part of the density box was wider than the width of the upstream, and the innermost density box contained the wake area of approximately 5 times the train height away from the nose of the tail car. The three density boxes from the inside to the outside were divided into a tetrahedral mesh with the grid sizes of $100 \mathrm{~mm}, 200 \mathrm{~mm}$, and $400 \mathrm{~mm}$, respectively. For each bogie area, the space was relatively narrow, and the volume 


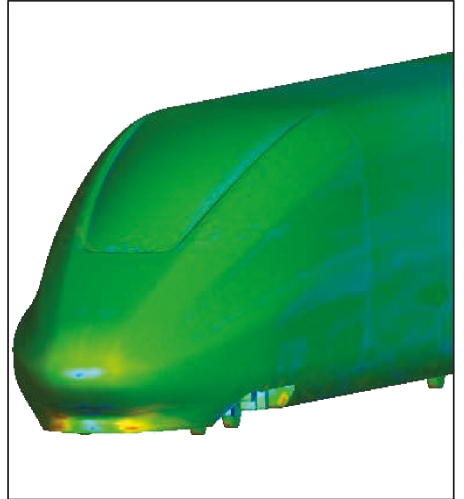

(a)

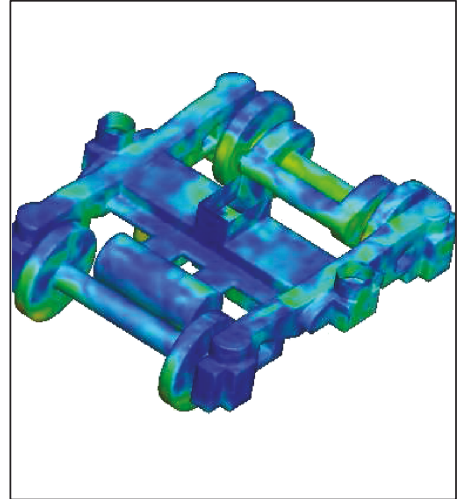

(b)

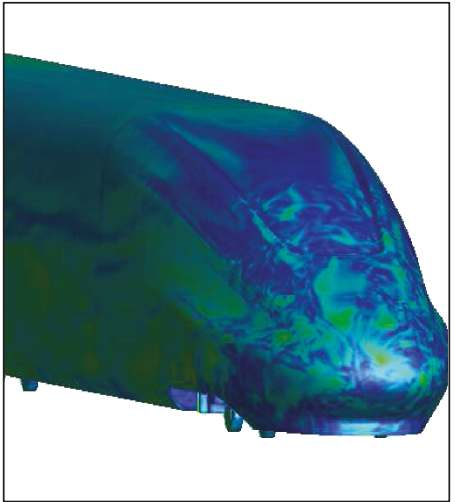

(c)

Y plus:

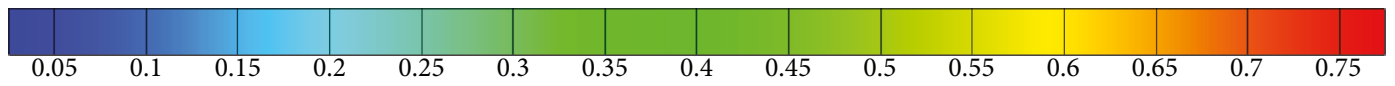

FIgURE 4: $Y$ plus (free flow speed: $97.22 \mathrm{~m} / \mathrm{s}$ ): (a) head car, (b) first bogie, and (c) tail car.

mesh distribution of this area can be controlled by the surface mesh. The thickness of the thin plates on both sides of the six bogie cavities was approximately $22.5 \mathrm{~mm}$. To accurately capture the overflow in those areas, the mesh size of $5 \mathrm{~mm}$ was adopted. The above mentioned sizes were all full scaled. The volume mesh number was approximately 85 million.

In general, the appropriate boundary layer grid distribution and the spatial grid distribution are two basic requirements for a high-precision LES.

The boundary layer grid distribution parameter is primarily the $y^{+}$[40], which is defined by (12). Because the LES needs to capture the coherent structure, the first information that triggers the coherent structure is the low-speed stripe structure formed at the viscous bottom layer $\left(y^{+}<10\right)$. Because the viscous sublayer is to be arranged with an appropriate number of grids, $y^{+}$should be less than one. Figure 4 shows $y^{+}$distribution on the train surface.

$$
y^{+}=\frac{\overline{U^{*}} y}{v},
$$

where $\overline{U^{*}}=\sqrt{\tau_{\omega} / \rho}$ is the wall friction velocity, $\tau_{\omega}$ is the wall shear stress, $y$ is the distance from the centroid of the firs layer of mesh to the wall, and $v$ is the kinematic viscosity coefficient.

The spatial distribution parameters of the grid are primarily grid scales. As the small-scale vortices have isotropic characteristics, the grid scales must be located in the local inertial subregion. Thus, the subgrid scale model established for small-scale vortices is reasonable. The integral scale is the criterion to distinguish the inertial subregion from the large-scale vortex region. Therefore, the grid scale should be smaller than the local integral scale. The local integral scale is defined as follows:

$$
l_{t}=\frac{C_{0} k^{3 / 2}}{\varepsilon}
$$

where $C_{0}=0.2, k$ is the turbulence energy, and $\varepsilon$ is the turbulence dissipation rate.

$$
\varepsilon=2 v_{t} \overline{\mathbf{S}}_{i j} \overline{\mathbf{S}}_{i j}
$$

where $v_{t}$ is the subgrid eddy viscosity coefficient, and $\mathbf{S}_{i j}$ is the strain rate tensor.

This article takes the $1 / 3$ power of the grid volume as the grid's equivalent length $l_{\Delta}$. The spatial distribution of $l_{\Delta} / l_{t}$ is shown in Figure 5.

Figures 4 and 5 show that the $Y$ plus on the train surface and the $l_{\Delta} / l_{t}$ in the fluid domain are both less than one. Consequently, the grid presented herein can meet the requirements for the accurate LES calculation.

The shear stress transport sst $k-\omega$ model was chosen to compute the steady flow field. The semi-implicit method for pressure-linked equation (SIMPLE) algorithm for the pressure-velocity coupling was used in the steady calculation. The continuity equation was discretized using the standard scheme. The momentum equation, turbulence kinetic energy equation, and specific turbulence dissipation rate equation were discretized using the second-order upwind scheme. The transient flow field computation utilized the Smagorinsky-Lilly-based LES turbulence model. The temporal difference equation used a second-order implicit scheme. The couple algorithm for the pressurevelocity coupling was used in the unsteady calculation. The momentum equation was discretized using a bounded central differencing scheme.

The transient time step $\Delta t$ was set to $5 \times 10^{-5} \mathrm{~s}$. According to the Nyquist sampling theorem, the corresponding noise frequency for analysis is $10 \mathrm{kHz}$. The time for the transient flow field computation was 6500 steps. The computation in the first 2500 steps was to ensure the full development of the turbulence flow field, and the remaining 4000 steps were to extract the noise source information. The sound source data in each time step was stored, with a time span of $0.2 \mathrm{~s}$ and a frequency resolution of $5 \mathrm{~Hz}$. 


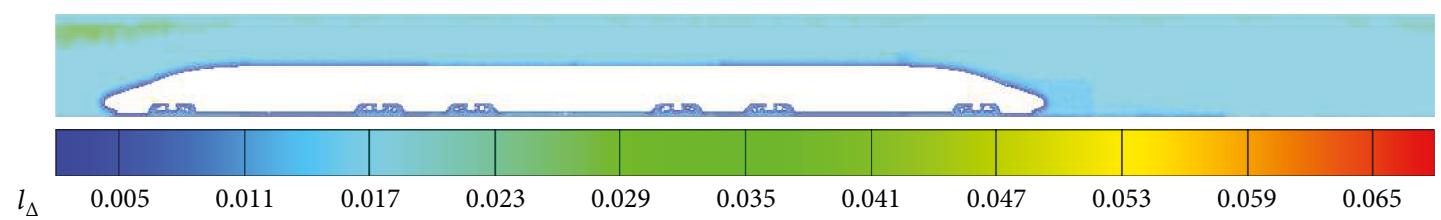

(a)

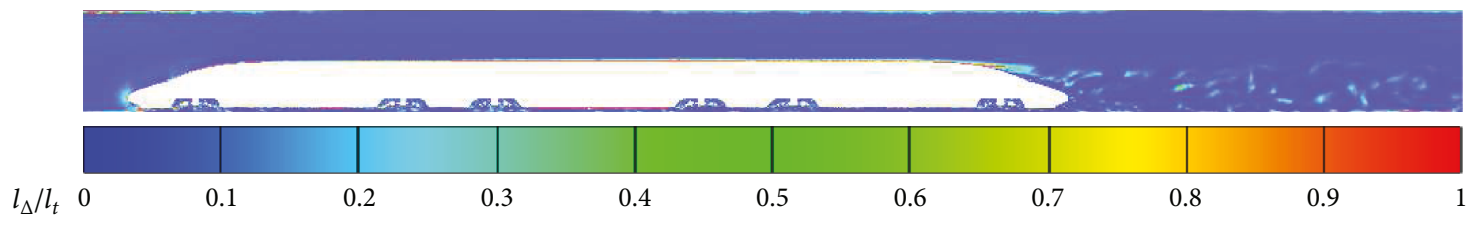

(b)

FIGURE 5: Grid parameters: (a) $l_{\Delta}$ (units: m) and (b) $l_{\Delta} / l_{t}$.

In this study, we used the Ansys Fluent software from the Beijing Computing Center to perform the numerical simulations.

3.2. Model Validation. Currently, we lack the test data of acoustic wind tunnel tests of high-speed train in tunnels because the current tests are aimed at open tracks. The rationality of the turbulence model and the meshing strategy adopted herein are verified by the acoustic wind tunnel test results of the high-speed train on the open tracks.

The aerodynamic noise test of a high-speed train [41] was performed at the opening test section of the $5.5 \mathrm{~m} \times 4 \mathrm{~m}$ acoustic wind tunnel in a research institute of China. The test model was a high-speed train with three cars at a scale of $1: 8$, which was installed on the floor with a railway bed. Figure 6 shows the aerodynamic noise test setup. Thirty far-field microphones were mounted on one side of the test model. The installation position is shown in Figure 7. In this study, the same mesh division strategy and turbulence model as defined in Section 3.1 were used to numerically simulate the fluctuating flow field around the high-speed trains, and the far-field radiated noise of the high-speed trains was predicted using Ffowcs Williams and Hawkings (FW-H) equation. The experimental results and simulation results of sound pressure levels at 30 receiving points are statistically averaged, and the spectrum curves of the mean sound pressure levels are plotted in Figure 8.

As shown in Figure 8, the simulation result and the test result have the same variation trend for the sound pressure level spectrum curve. Especially when the frequency is below $2 \mathrm{kHz}$, the simulation results of the sound pressure level compare well with the test results. However, the degree of coincidence between the simulation results and the test results is relatively poor when the frequency is above $2 \mathrm{kHz}$, but the difference does not exceed $2 \mathrm{~dB}$. The primary reason is that the computational grid herein did not accurately capture the fluctuating flow field that resulted in frequencies above $2 \mathrm{kHz}$. However, the high-speed trains' aerodynamic source primarily distributes below $2 \mathrm{kHz}$. In conclusion, the turbulence model, mesh generation strategy, and nonreflective boundary conditions adopted in this paper meet the

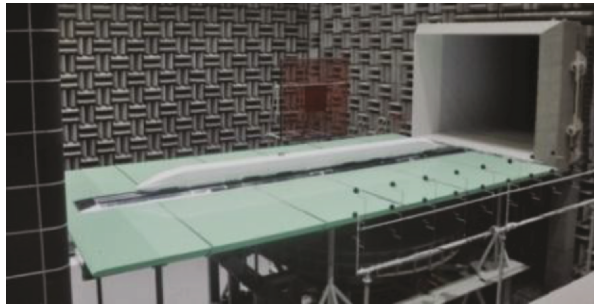

Figure 6: Acoustic wind tunnel test site 0 .

requirements of refined numerical simulation and can provide strong support for this study.

\section{Flow Field Structure}

Figures 9 and 10, respectively, show the instantaneous magnitude of the airflow velocity/vortex spatial distribution contour around the high-speed train in tunnels. Figure 11 shows the spatial distribution of instantaneous vortex structures of high-speed trains in tunnels. The vortex structures are identified by the $Q$ criterion [42] and use the contour level of Figure 10. In Figures 9-11, the contour maps indicated by the arrows are all the partially enlarged views.

According to the structure characteristics and the region of the flow field, the flow structures around the high-speed train in the tunnel are divided into six types, which are shown by the Rome digital mark in Figure 11.

The first type of flow field structure is located in the front windshield of the head car. In this area, it is evident that when the airflow passes through the recess of the front windshield, the velocity magnitude rapidly decreases, the vorticity magnitude rapidly increases, and the vortex structures fall off periodically. A list of vortex structures traverses downstream along the front windshield, and the other two lists of vortex structures traverse downstream along the side windows. As the thickness of the boundary layer increases, the distance between the shed vortex structures gradually decreases.

The second type of flow field structure is located in the head car cowcatcher. It is evident that the air flowing down 


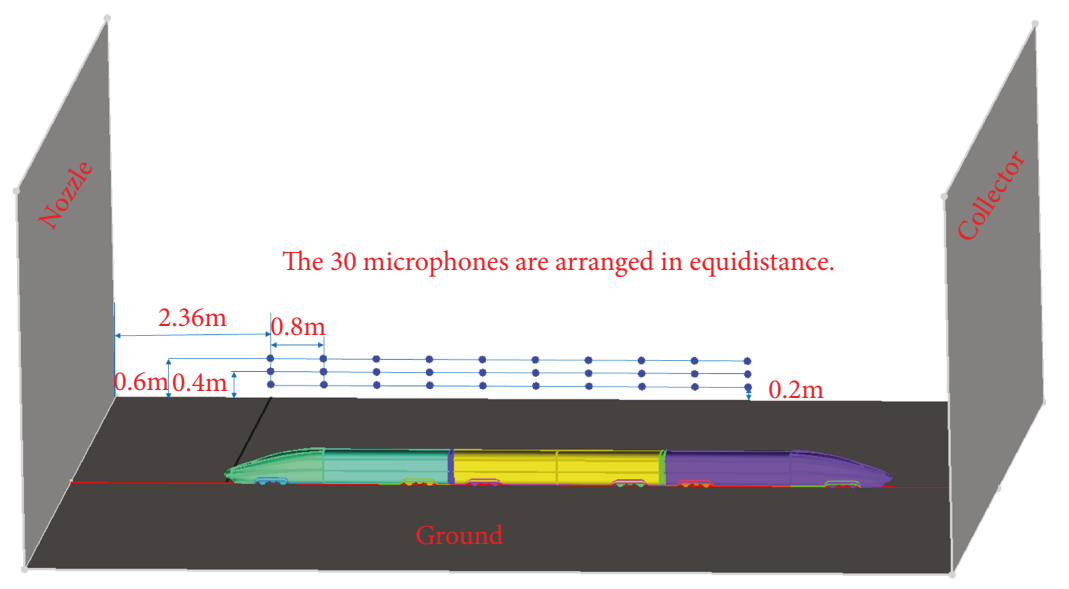

FIgURE 7: Thirty far-field microphone positions.

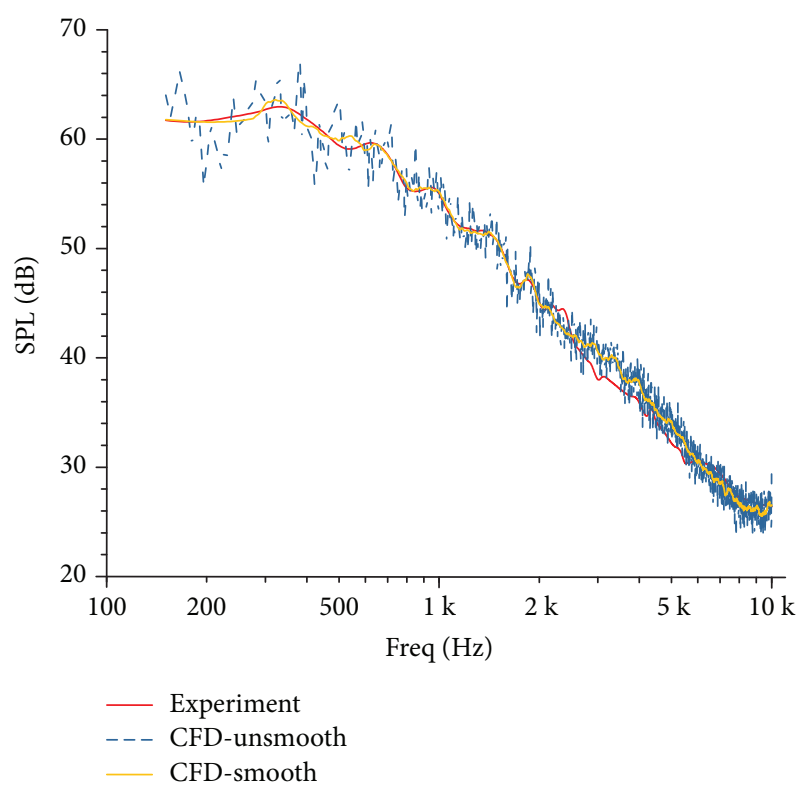

Figure 8: Comparison of simulation results with acoustic wind tunnel test results $0(55.56 \mathrm{~m} / \mathrm{s})$.

from the nose tip and the airflow downstream are mixed in the recesses of the head car cowcatcher. The velocity magnitude decreases rapidly, and the vorticity magnitude increases rapidly. Subsequently, this airflow bypasses the cowcatcher's cusp. Owing to the effect of the turbulent boundary layer of the car body, the velocity of this airflow decreases further, and the vorticity amplitude increases further. The low velocity and strong vorticity area is formed in the narrow space around the head car's cowcatcher. When the downstream airflow flows through the under-car space near the head car's cowcatcher, the airflow accelerates in this area because of the space reduction. However, the vorticity magnitude is relatively small because the ground is a moving wall boundary, forming a high-speed and low-vortex area near the ground. Two lists of largerscale one-pin hairpin vortices traverse along both sides of the cowcatcher to the downstream bogie 01 cavity, and a large number of small-scale hairpin vortices envelop the head car cowcatcher to form a large area of the vortex pack.

The third type of flow field structure is located in bogie 01. As shown, when the upstream shear layer airflow flows through the guide edge of the bogie cavity, because of the geometrical discontinuity, this shear layer airflow is divided into two airflows. A stream of air flows down the stream, passes through the rear wheel area of the bogie, and hits the rear of the bogie cavity. The other airflow traverses along the front of the bogie cavity into the interior of the cavity. Owing to the disturbing effect of the bogie, the shear flow is hit into a broken shape. Although the bogie structure contains cylindrical rods, the Reynolds number of the rod reaches $10^{5}$ orders of magnitude; further, the shed vortex of the rod and the generated vortex of the cavity interfere with each other such that the bogie structure cannot generate an obvious vortex street structure. A large number of small hairpin vortices are generated inside the cavity, and larger-scale one-pin hairpin vortices are generated outside the cavity. The velocity/vorticity magnitude distribution in this area is rather uneven. The vorticity magnitude of the rear wheel set region and the downstream shear layer airflow's passing region are relatively large; however, the vorticity magnitude of the region underneath this shear layer airflow and the front wheelset region are significantly smaller. The flow field structure in other bogies has similar results, but the change in the flow field is not as violent as that in the bogie 01 area.

The fourth type of flow field structure is located in both sides of the tail car. In this area, the air flows down from the tail streamlined shoulder, and the overflows from the bogie $05 /$ bogie 06 and the downstream airflow from both sides of the body are strongly mixed. The velocity magnitude decreases and the vorticity magnitude increases. The hairpin vortex generated in this area merges with the hairpin vortex that overflows from both sides of the bogie 05/06 cavity, forming a strong fluctuating turbulence.

The fifth type of flow field structure is located in the area of the tail car front windshield. In this area, a clear separation exists of the airflow at the tail streamlined 


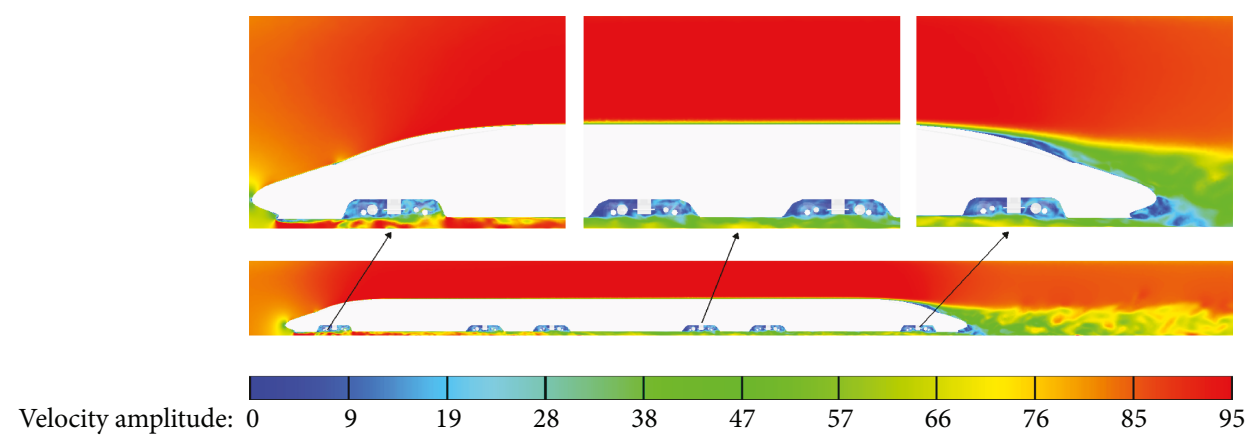

(a)

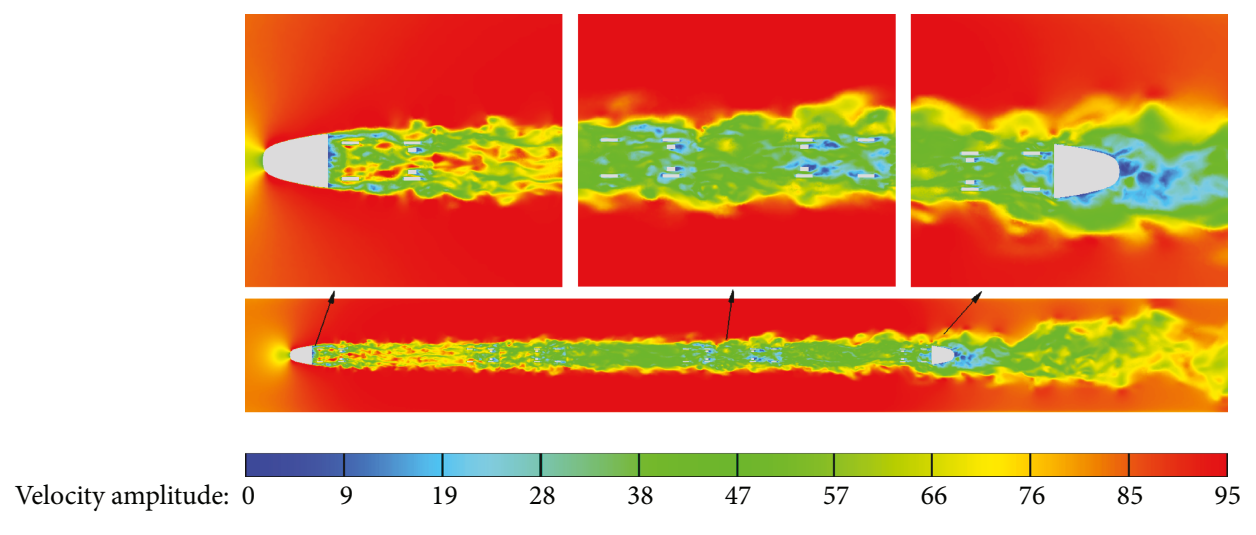

(b)

FIGURE 9: Velocity amplitude (units: $\mathrm{m} / \mathrm{s}$; free flow speed: $83 \mathrm{~m} / \mathrm{s}$ ): (a) longitudinal symmetry plane of the train and (b) $x-y$ plane that is $0.1 \mathrm{~m}$ above the ground.

shoulder. The airflow is blocked at the downstream recess of the tail car front windshield, resulting in a smaller air velocity in the area and a small-scale hairpin vortex. The large-scale one-pin hairpin vorticity at both sides of the front windshield begins to fall off and traverses downstream along the two sides, combining with the hairpin vortex generated downstream of the front windshield and generating a strong fluctuating pressure at both sides of the front windshield.

The sixth type of flow field structure is located in the wake area. In the wake area, which is approximately five times the train height away from the nose of the tail car, the vorticity magnitude is relatively large, but the velocity magnitude is relatively small. In particular, the velocity magnitude around the tail car cowcatcher is close to zero, and the vorticity magnitude is one order of magnitude larger than that of the other wake regions. The isosurface of the vorticity/velocity magnitude is wavy in this area, and different isosurfaces are nested with each other, resulting in a disordered distribution of the vorticity/velocity. The vortex structure in the wake area is primarily the one-pin hairpin vortex group. Although the vortex structure's distribution is disorderly and unsystematic, the vortex feet of the hairpin vortex point to both sides. The upper hairpin vortex group is primarily from the upper of the tail car streamlined part with the large-scale and weak vorticity. The lower hairpin vortex group is primarily from the bottom of the tail car with the small-scale and strong vorticity. When the hairpin vortex group traverses downstream, the width of the hairpin vortex group gradually increases, and the length of the vortex feet of the hairpin vortex is gradually elongated to a certain extent and subsequently broken into small-scale hairpin vortices.

The scale of the vortex structure is closely related to the frequency of the aerodynamic noise. In general, the larger the scale of the vortex structure, the lower is the sound frequency. Figure 11(b) indicates five typical vortex structure scales: $40 \mathrm{~mm}, 180 \mathrm{~mm}, 80 \mathrm{~mm}, 220 \mathrm{~mm}$, and $145 \mathrm{~mm}$. By comparing with the characteristic scales of the five typical vortex structures, the scale range of the vortex structure captured is approximately $(10-500 \mathrm{~mm})$ in the whole computing domain, (10-40 mm) within the bogie cavity, and (100-500 $\mathrm{mm})$ in the wake area.

The six regions above have different flow field structure characteristics and different formation mechanisms, but they all generate strong fluctuating flow fields.

\section{Aerodynamic Noise Source}

It is known from Section 2.3 that the rate of change of the fluctuating pressure $p^{\prime}$ on the high-speed train can characterize the intensity of its aerodynamic noise source. This section uses $P^{\prime}$ on the high-speed train to quantitatively study the contribution rate, frequency characteristics, and velocity dependence law of sound energy of each component. The 


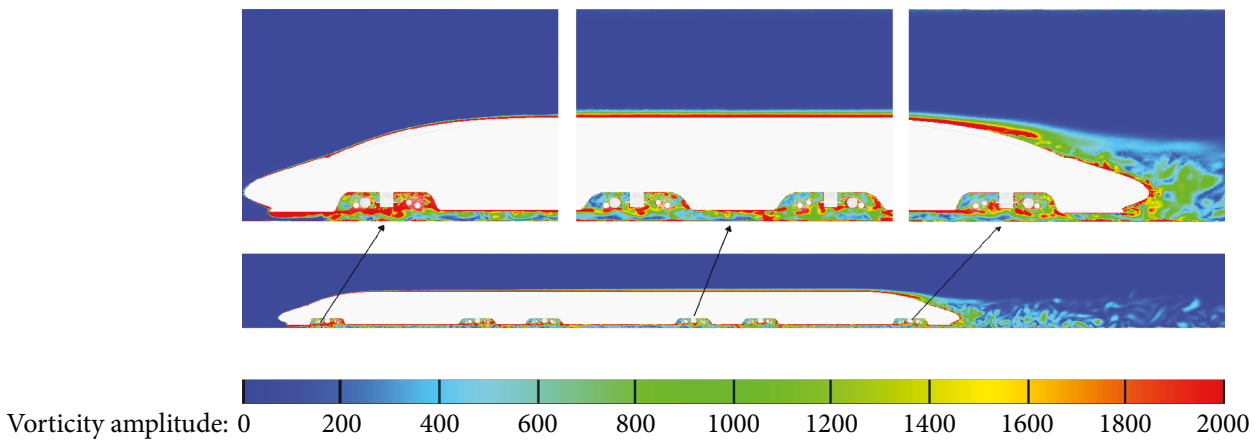

(a)
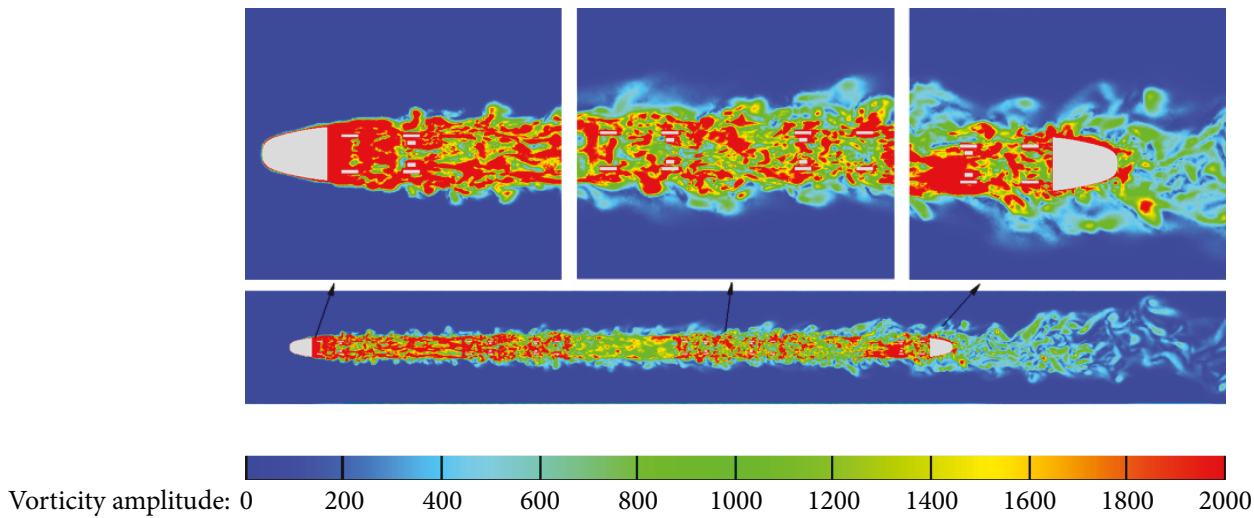

(b)

FIGURE 10: Vorticity amplitude (units: $1 / \mathrm{s}$; free flow speed: $83 \mathrm{~m} / \mathrm{s}$ ): (a) longitudinal symmetry plane of the train and (b) $x-y$ plane that is $0.1 \mathrm{~m}$ above the ground.

data for the surface-fluctuating pressure was processed in five steps. The first step was to extract the time domain signal of the fluctuating pressure $p$ on the high-speed train. The second step was to perform the Fourier transform and conversion to $1 / 3$ octave bands on $p$ and obtain $P$. The third step was to obtain the fluctuating pressure change rate $P^{\prime}$ according to the Fourier transform property of the derivative, i.e., (15). The fourth step was to calculate the rate of change of the fluctuating force $F^{\prime}$ via (16). The fifth step was to calculate the equivalent acoustic power of the sound source $W_{\text {source }}$ via (11).

$$
\begin{aligned}
& P^{\prime}=2 \pi f P, \\
& F^{\prime}=\int_{s} P^{\prime} d s,
\end{aligned}
$$

where $p$ is the fluctuating pressure on the high-speed train, in units of $\mathrm{Pa} ; P$ is the physical quantity of the Fourier transform for $p$, in units of $\mathrm{Pa}$; $f$ is the frequency, in units of $\mathrm{Hz}$; $F$ is the fluctuating force, in units of $\mathrm{N}$; $s$ is the area of the noise source, in units of $\mathrm{m}^{2}$; the right superscript ' represents the time derivative.

5.1. Intensity and Frequency Characteristics. The mean square root of the rate of fluctuating pressure (dpdt-rms) on the high-speed train is defined by (17) and characterizes the average effect of the sound source intensity in the sampling time. Its distribution cloud is shown in Figure 12.

$$
p_{\mathrm{rms}}^{\prime}=\left[\frac{1}{T} \int_{0}^{T}\left(p^{\prime}\right)^{2} \mathbf{d} t\right]^{1 / 2},
$$

where $T$ is the sampling time.

Figure 12 shows that the areas with the strong intensity of the noise source on the high-speed train are primarily located around the head car's cowcatcher, the recess of the front windshield on the head car, the bogies, and both sides of the tail streamlined part. The noise source strength of bogie 01 is obviously stronger than that of other bogies, and the noise source strength of the bogie's trailing edge is obviously stronger than that of its guide edge. It is noteworthy that the noise source intensity on the downstream two sides of the bogie cavity is significantly stronger than that on its upstream two sides. These results are consistent with the results of Iglesias et al. and Yamazaki and Ido.

Figure 13 illustrates the percentage of sound source energy from each component of the high-speed train and different partitioned areas in the total sound source energy. The serial number of each component in Figure 13(a) is the same as that in Figure 1. In Figure 13(b), partition strategy I is to divide the upside of the body into the upside of the head car (including the upside of the head streamlined part and 


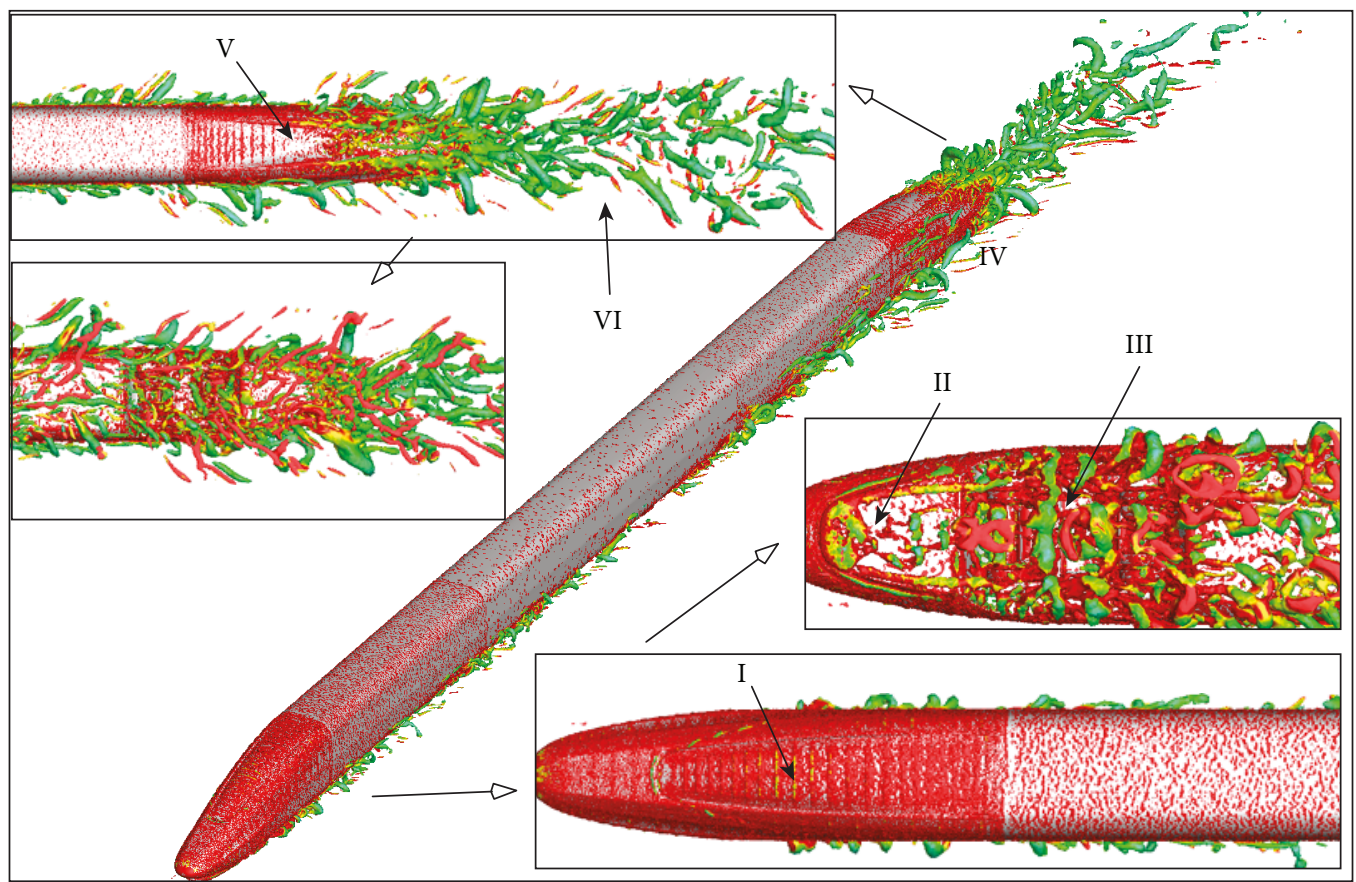

(a)

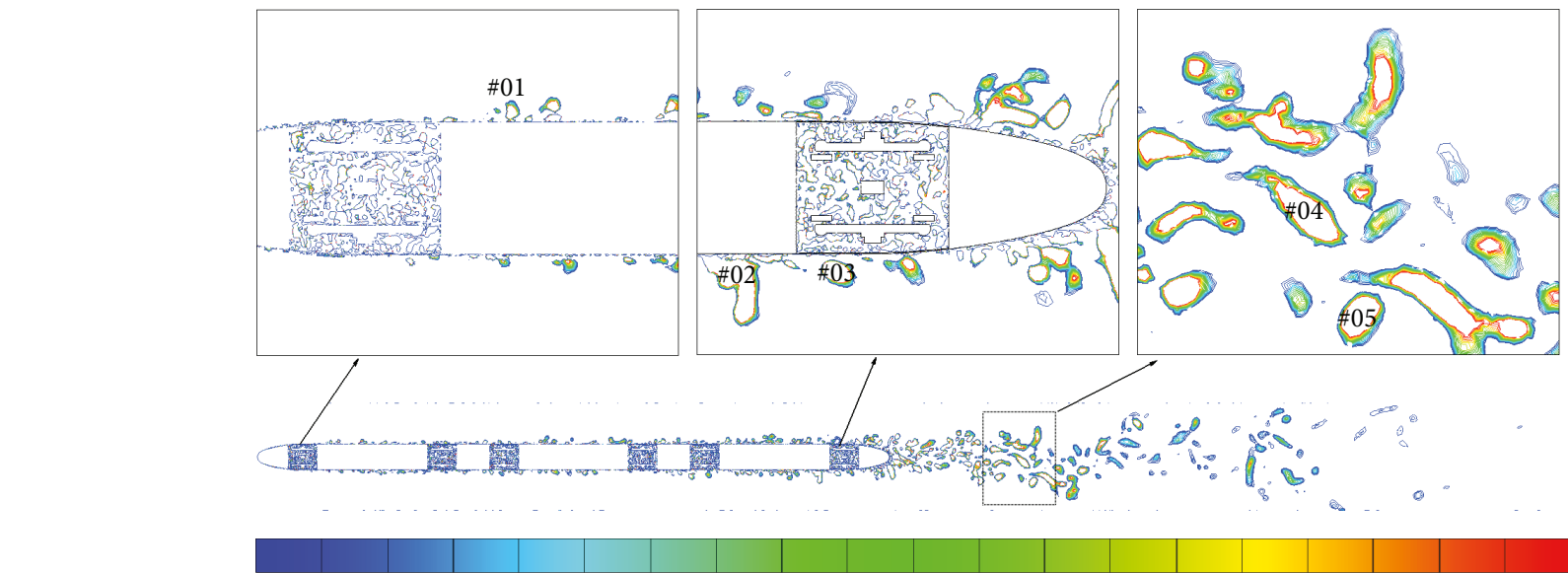

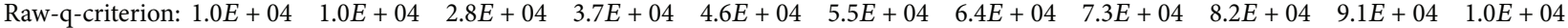

(b)

FIGURE 11: Vortex structure (free flow speed: $83 \mathrm{~m} / \mathrm{s}$ ): (a) instantaneous isosurface plot of $Q=200,000 / \mathrm{s}^{2}$, colored by vorticity and (b) contour map of $Q$ value on the $x-y$ surface that is $0.125 \mathrm{~m}$ above the ground.

the upside of the head coach), the upside of the middle coach, and the upside of the tail car (including the upside of the tail streamlined part and the upside of the head coach); partition strategy II is to divide the downside of the body into the downside of the head car (including the downside of the head streamlined part, the downside of the head coach, the first/ second bogie), the downside of the middle coach (including the downside of the middle coach, the third/fourth bogie), and the downside of the tail car (including the downside of the tail streamlined part, the downside of the tail coach and the fifth/sixth bogie); partition strategy III is to divide the whole car into the head car (including the upside/downside of the head streamlined part, the upside/downside of the head coach, the first/second bogie), the middle car (including the upside/downside of the middle coach, the third/fourth bogie), and the tail car (including the upper/downside of the tail streamlined part, the upper/downside of the tail coach, and the fifth/sixth bogie).

Based on Figure 13, the following conclusion is obtained. On the upside of the high-speed train, the sound energy of the upstream coach is less than that of the downstream coach, and its sound energy accounts for approximately $44.4 \%$ of the total sound energy. On the downside of the high-speed train, the sound energy of the upstream coach is more than that of the downstream coach, and its sound energy accounts for approximately $55.6 \%$ of the total sound energy. On the whole car, the sound energy of the upstream coach is more than that of the downstream coach, and the 

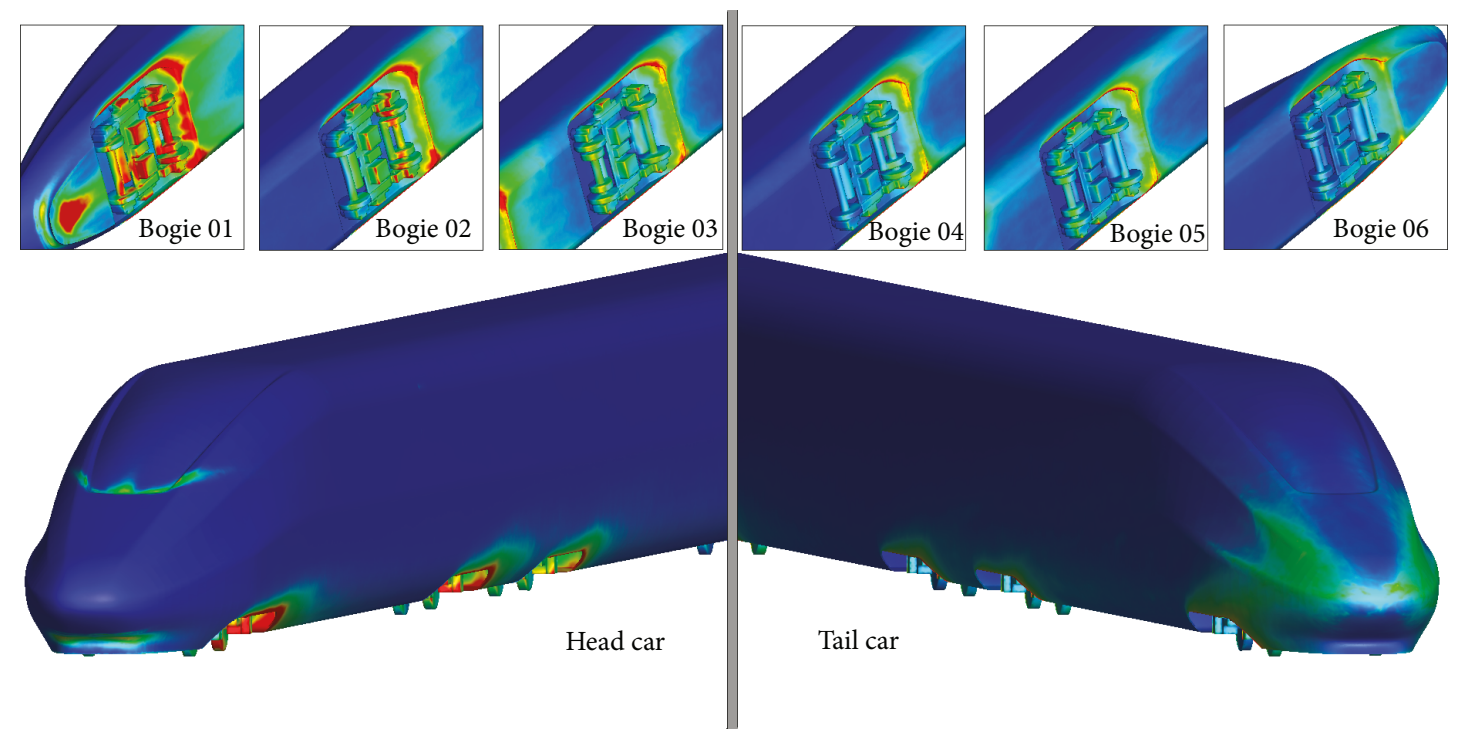

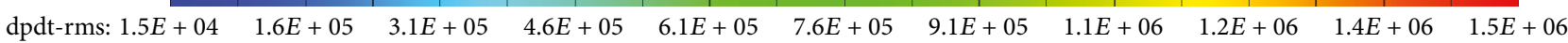

FIGURE 12: Contour of $p_{\text {rms }}{ }^{\prime}$ on the high-speed train (units: $\mathrm{Pa} / \mathrm{s}$; free flow speed: $83 \mathrm{~m} / \mathrm{s}$ ).

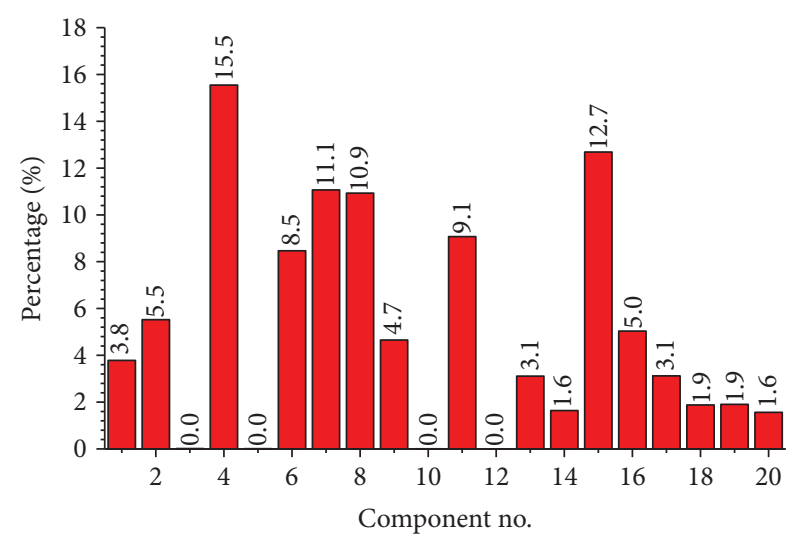

(a)

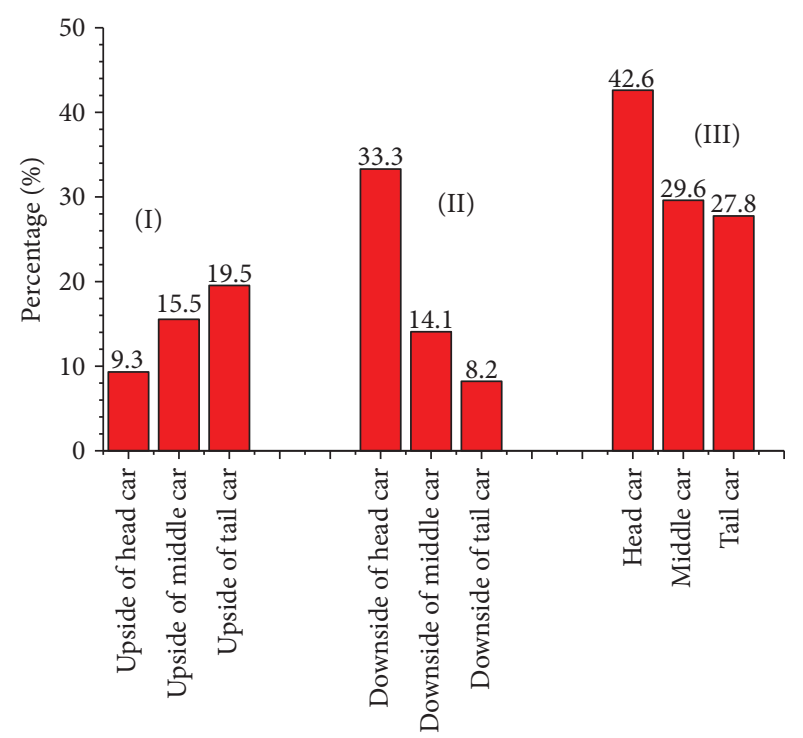

(b)

FIGURE 13: Percentage of sound source energy (flow speed: $83 \mathrm{~m} / \mathrm{s}$ ): (a) components and (b) train regions.

sound source energy of the head car accounts for approximately $42.6 \%$ of the total sound energy. On the same coach, the ratio of the sound energy is approximately $1: 3.57$ between the upside and downside of the head car, 1.11:1 between the upside and downside of the middle car, and 2.38:1 between the upside and downside of the tail car. On the six bogies, the sound source energy of the upstream bogie is greater than that of the downstream bogie, but the sound source energy of the tail bogie exhibits a local increase. The sound source energy of the six bogies accounts for approximately $26.2 \%$ of the total energy. It is noteworthy that the upper part of the mid-car contributes the most to the aerodynamic noise energy of the whole train, and the bottom of the mid-car contributes significantly to the aerodynamic noise energy of the whole train. This is because the area of these two components is significantly larger than that of other components, and the component is in the piston acceleration zone, and its fluctuating intensity is relatively significant.

Owing to space constraints herein, only the distribution contours of $P^{\prime}$ on the high-speed train at six typical frequencies are shown in Figure 14. Figures 14(a) and 14(b) show the distribution characteristics of $P^{\prime}$ at low frequencies. Figures 14(c) and 14(d) show the distribution characteristics 


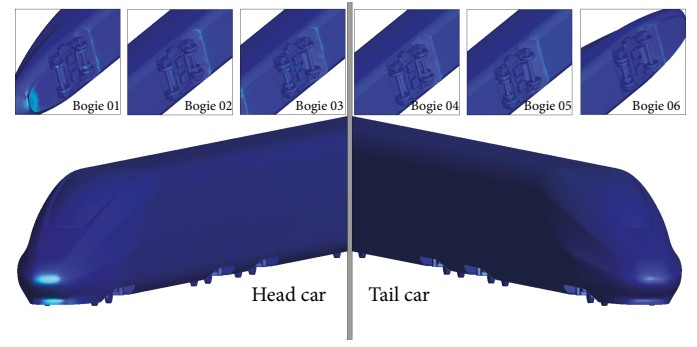

(a)

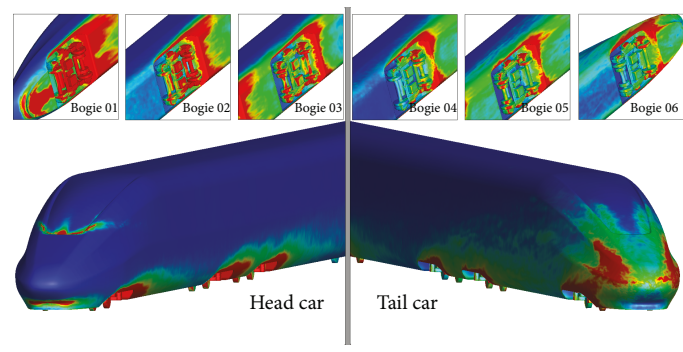

(c)

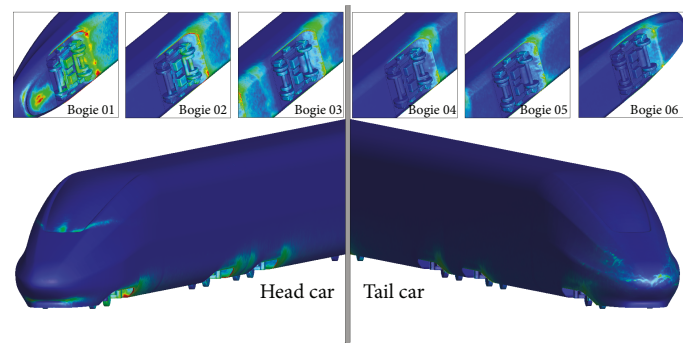

(e)

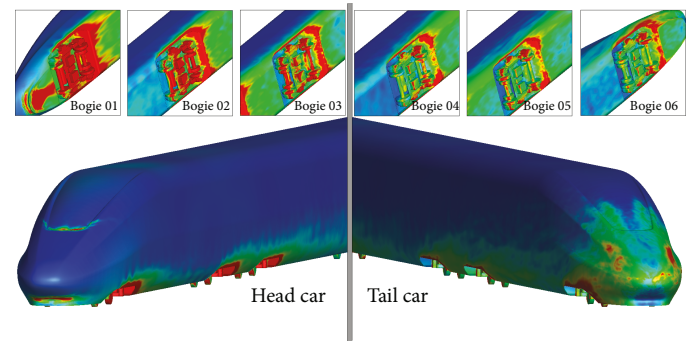

(b)

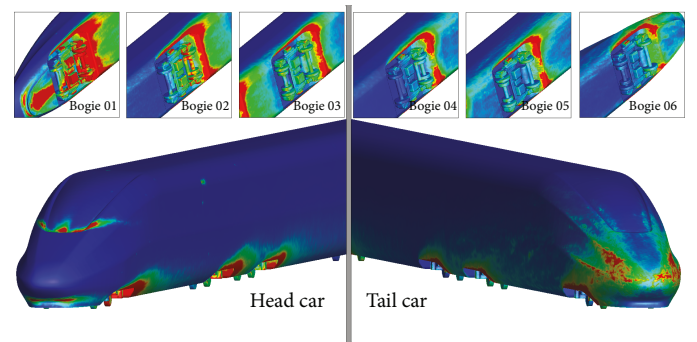

(d)

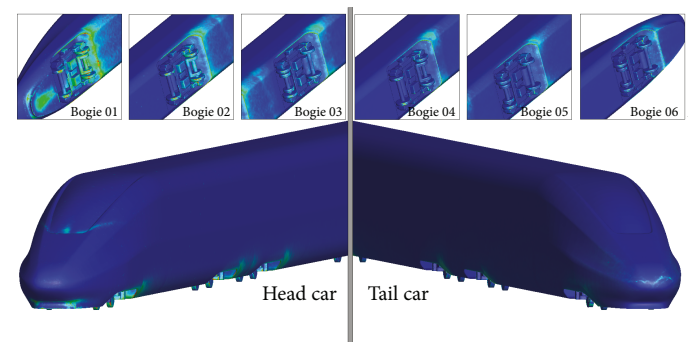

(f)

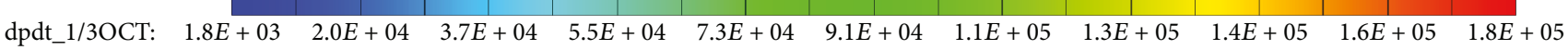

Figure 14: Contour of $P^{\prime}$ on the high-speed train (units: Pa/s; free flow speed: $83 \mathrm{~m} / \mathrm{s}$ ): (a) $16 \mathrm{~Hz}$, (b) $400 \mathrm{~Hz}$, (c) $800 \mathrm{~Hz}$, (d) $2 \mathrm{kHz}$, (e) $6.3 \mathrm{kHz}$, and (f) $10 \mathrm{kHz}$.

of $P^{\prime}$ at intermediate frequencies. Figures $14(\mathrm{e})$ and $14(\mathrm{f})$ show the distribution characteristics of $P^{\prime}$ at high frequencies. The frequency characteristics of sound source studied in this paper are all aimed at 1:8 scaled model.

According to Figure 14, on the head car's nose, the sound source intensity at $16 \mathrm{~Hz}$ is relatively strong while negligible at other frequencies, indicating that the sound source energy on the head car's nose is in the low-frequency range; on the head/trail car's cowcatcher, the recesses of the front windshield on the head car, the bogies, and both sides of the tail streamlined part, the sound source intensities at $400 \mathrm{~Hz}$, $800 \mathrm{~Hz}$, and $2 \mathrm{kHz}$, are relatively strong while weak at other frequencies, indicating that the sound source energy on these areas is in the middle-frequency range. To more clearly identify the frequency characteristics of the sound source energy, Figure 15 shows the percentage of $W_{\text {source }}$ from the whole car with respect to the total sound source energy at each frequency band according to the $1 / 3$ octave. The analysis frequency range is $(16-10 \mathrm{kHz})$.

According to Figure 15, the sound energy spectrum of the whole car presents broadband and multipeak characteristics.

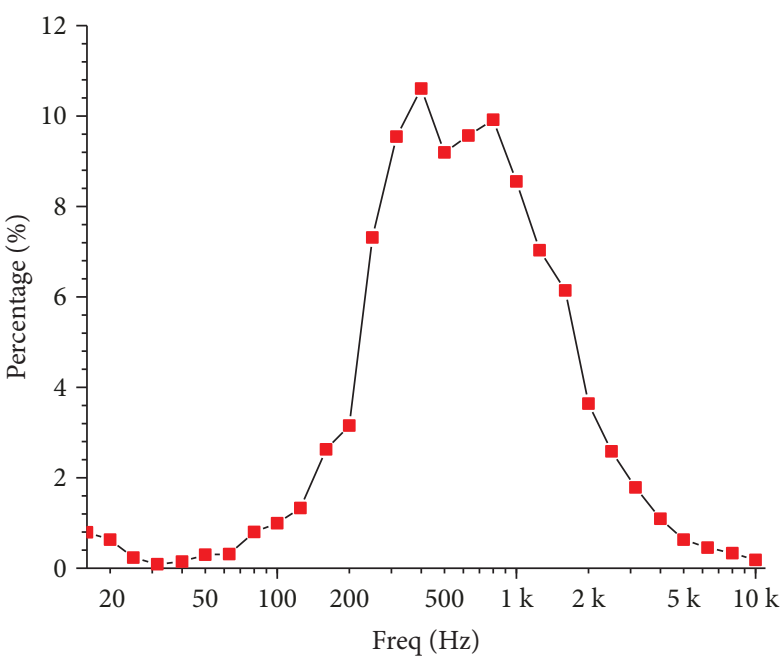

FIgURE 15: Percentage curve for the sound source energy at each frequency band (free flow speed: $83 \mathrm{~m} / \mathrm{s}$ ). 


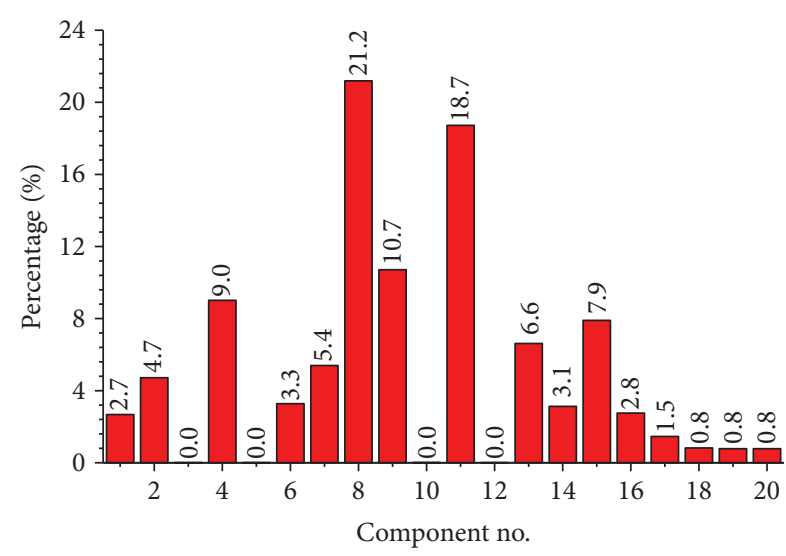

(a)

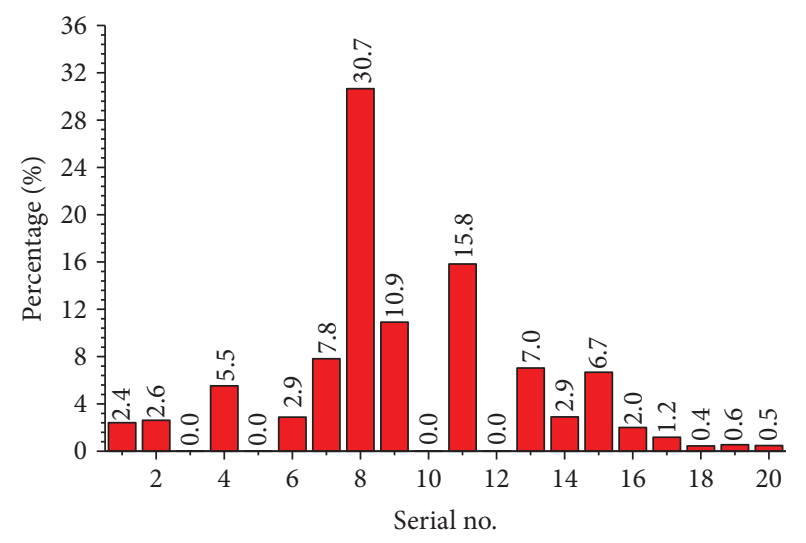

(b)

Figure 16: Contribution rate histogram (free flow speed: $83 \mathrm{~m} / \mathrm{s}$ ): (a) $400 \mathrm{~Hz}$ and (b) $800 \mathrm{~Hz}$.

The dominant distribution frequency range is from $100 \mathrm{~Hz}$ to $4 \mathrm{kHz}$, accounting for approximately $95.1 \%$ of the total sound source energy. The peak frequencies are $400 \mathrm{~Hz}$ and $800 \mathrm{~Hz}$. Combined with the sound source intensity distribution shown in Figure 16(a), we found that the dominant contribution to the sound source energy at $16 \mathrm{~Hz}$ of the whole car is from the nose of the head car. To clearly identify the dominant contribution components of the total sound source energies at $400 \mathrm{~Hz}$ and $800 \mathrm{~Hz}$, the sound source energy of each component at $400 \mathrm{~Hz}$ and $800 \mathrm{~Hz}$ is divided by the total sound source energy at $400 \mathrm{~Hz}$ and $800 \mathrm{~Hz}$, respectively. The statistical results are shown in Figure 16. The number of components in Figure 16 is shown in Figure 1.

According to Figure 16, the largest contributors to the total sound source energy at $400 \mathrm{~Hz}$ and $800 \mathrm{~Hz}$ are both the downside of the head streamlined part, followed by the downside of the middle car. The contribution rate of all the components at the downside of the train is, respectively, $74.9 \%$ and $78.7 \%$ to the total sound source energy at $400 \mathrm{~Hz}$ and $800 \mathrm{~Hz}$, of which six bogies contribute $14.5 \%$ and $11.4 \%$, respectively. To further analyze the spectrum characteristics of the sound source energy for the bottom of the head car's streamlined part, the percentage of the sound source energy at each $1 / 3$ octave frequency band to the whole frequency range in this area is calculated. The statistical results are shown in Figure 17.

According to Figure 17, the sound source energy spectrum of the bottom of the head car's streamlined part presents broadband and multipeak characteristics. The dominant distribution frequency range is from $200 \mathrm{~Hz}$ to $2 \mathrm{kHz}$, accounting for approximately $89.4 \%$ of the total sound source energy of this component. The peak frequencies are $400 \mathrm{~Hz}$ and $800 \mathrm{~Hz}$.

In summary, when the incoming flow velocity is $83 \mathrm{~m} / \mathrm{s}$, the dominant noise source of the high-speed train is from the downside of the train. The sound source energy of the downside of the head car is much higher than that at the downside of the other coaches. The dominant frequency distribution range is from $200 \mathrm{~Hz}$ to $2 \mathrm{kHz}$. The dominant peak frequency includes $400 \mathrm{~Hz}$ and $800 \mathrm{~Hz}$. The dominant

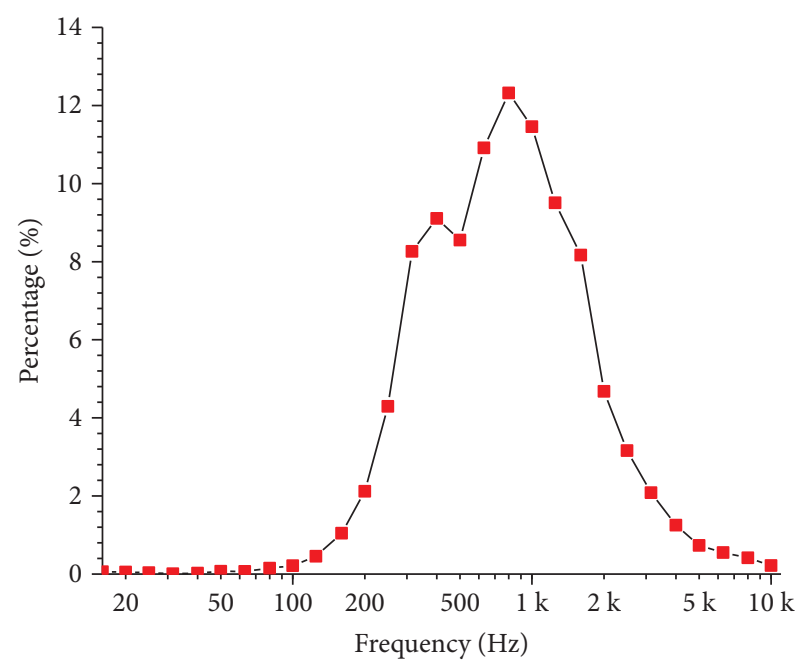

Figure 17: Percentage curve for the sound source energy at each frequency band on the bottom of the head car's streamlined part (component 8, free flow speed: $83 \mathrm{~m} / \mathrm{s}$ ).

sound sources of the downside of the train at $400 \mathrm{~Hz}$ and $800 \mathrm{~Hz}$ are both from the bogie cavity, rather than the bogie.

5.2. Inflow Velocity Dependence Regularity. Figure 18 fits the functional relationship between the sound source energy and the inflow velocity for the whole car and the dominant sound sources. The sound source energy of the corresponding research object at the inflow speed of $50 \mathrm{~m} / \mathrm{s}$ was the dimensionless reference variable. The fitting function is $y=a x^{b}$. The fitting results are shown in Table 1.

According to Figure 18 and Table 1, the sound source energy of the whole car, the bottom of the head car's streamlined part, and bogie 01 follows an $n$-power relations with the inflow velocity and $n$ being closer to six. The sound source intensities of the monopole, dipole, and quadrupole are proportional to the 4th, 6th, and 8th power of the inflow velocity, respectively. Consequently, the aerodynamic noise source of the high-speed train presents the characteristics of 


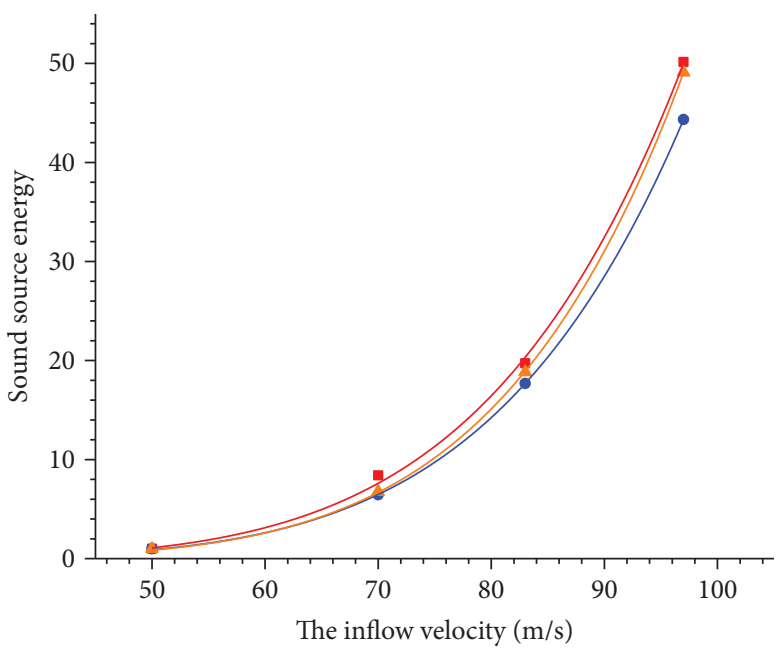
- Sample data of the whole car — Fitting curve of the whole car
- Sample data of the bottom of - Fitting curve of the bottom of the head car's streamlined part the head car's streamlined part
$\Delta$ Sample of bogie $01 \quad$ Fitting curve of bogie 01

FIGURE 18: Functional relationship between the sound source energy and the inflow velocity.

TABLe 1: Fitting coefficients between the sound source energy and the inflow velocity.

\begin{tabular}{|c|c|c|c|c|c|}
\hline \multirow{2}{*}{ Area } & \multicolumn{2}{|c|}{$a$} & \multicolumn{2}{|c|}{$b$} & \multirow{2}{*}{ Fitting degree } \\
\hline & Fitting coefficient & Standard deviation & Fitting coefficient & Standard deviation & \\
\hline The whole car & $1.69 E-10$ & $1.473 E-10$ & 5.77 & 0.191 & 0.99888 \\
\hline $\begin{array}{l}\text { The bottom of the head car's } \\
\text { streamlined part }\end{array}$ & $8.57 E-11$ & $9.701 E-12$ & 5.90 & 0.025 & 0.99998 \\
\hline Bogie 01 & $3.50 E-11$ & $6.295 E-12$ & 6.11 & 0.040 & 0.99996 \\
\hline
\end{tabular}

the dipole noise source when the inflow velocity is in the range of $(50-97 \mathrm{~m} / \mathrm{s})$.

In general, the sound source energy spectrum shows the frequency similarity for the cavity. The downside of the train is composed of the cavity and the bogie. Whether the sound source energy spectrum shows the frequency similarity for the downside of the train is to be determined.

Using the bottom of the head streamlined part as an example, the sound source energy at each frequency according to the $1 / 3$ octave is divided by the total energy of the corresponding source at the corresponding flow velocity. The percentage of the sound source energy of this area is obtained at each frequency, as shown in Figure 19.

Figure 19 shows that the sound source energy spectrum of the bottom of the head streamlined part shows a strong frequency similarity, which is consistent with the results of the tests conducted by Andreas Lauterbach in acoustic wind tunnels. This further demonstrates the accuracy of the simulation results herein.

In summary, when the inflow velocity is in the range of $(50-97 \mathrm{~m} / \mathrm{s})$, the aerodynamic noise source energy of the high-speed train is proportional to the 6th power of the inflow velocity, which shows the characteristics of the dipole noise source. The sound source spectrum of the bottom of the head streamlined part has frequency similarity.

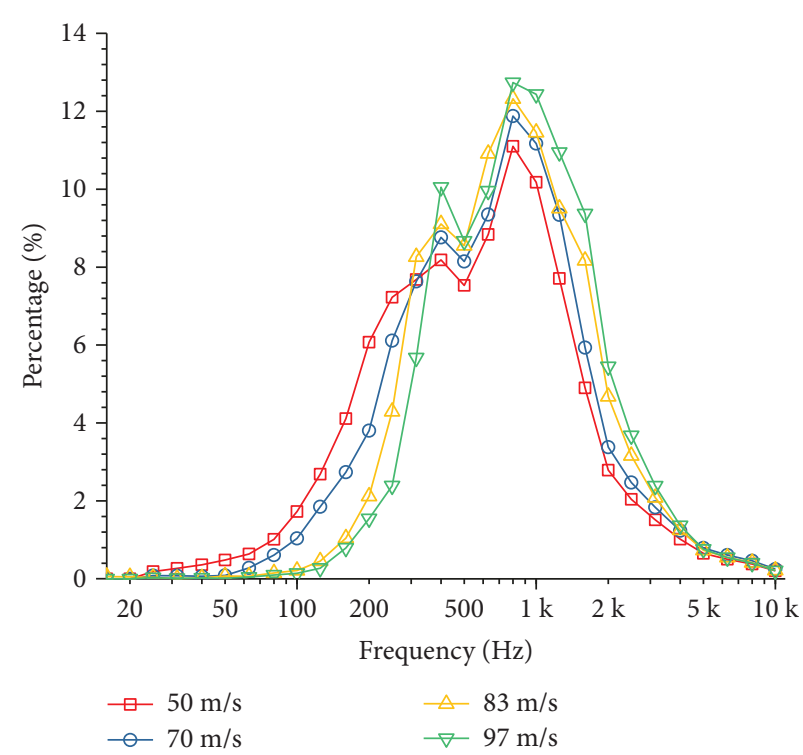

FIGURE 19: Sound source energy spectrum of the bottom of the head streamlined part (component 8), at different inflow velocities. 


\section{Noise Mechanism Analysis}

According to the results of Section 4 and Section 5, the dominant sound source on the high-speed train is located in the low/high velocity and strong vorticity area, while the low/high velocity and strong vorticity area is the vortex structure gathering area. The intensity of the sound source and the vortex structure around the bogie cavity's trailing edge are stronger than those around its leading edge. The sound source energy and variation in flow field around bogie 01 are much larger than that around the other bogies. The sound source intensity on two downstream sides of the bogie cavity is stronger than that on its two upstream sides. The vortex structure overflowing from both sides of the bogie cavity reacts on its two sides. Therefore, we conclude that the stronger the vortex structure and the wider the area of the strong vortex, the stronger is the intensity of the sound source and the wider is the scope of the stronger sound source area.

Several conclusions can be drawn from the results in Section 5. The dominant sound sources of the train's downside at $400 \mathrm{~Hz}$ and $800 \mathrm{~Hz}$ are the bogie cavity, rather than the bogie. The sound source energy's spectral pattern of the bottom of the head streamlined part has a frequency similarity, similar to that of the cavity sound source. The train's downside contains six cavities. Therefore, a preliminary conclusion can be obtained: the cavity sound mode dominates the mechanism of the aerodynamic sound source of the train's downside at $400 \mathrm{~Hz}$ and $800 \mathrm{~Hz}$. To further verify this conclusion, we will provide a discussion below using the sound theory of the flow in the cavity.

The bogie cavity is approximately $4.2 \mathrm{~m}$ long, $2.9 \mathrm{~m}$ wide, and $0.8 \mathrm{~m}$ deep. According to Rossiter's semiempirical formula for the model frequency of the cavity fluid dynamic oscillation 0 , as shown in (17), the first-, fifth-, and tenthorder model frequencies are approximately $60 \mathrm{~Hz}, 380 \mathrm{~Hz}$, and $780 \mathrm{~Hz}$, respectively. According to the formula for the resonant frequency of the cavity standing wave 0 , as shown in (18), the first- and the second-order resonant frequencies are approximately $260 \mathrm{~Hz}$ and $780 \mathrm{~Hz}$, respectively. We found that the tenth-order hydrodynamic oscillation mode frequency is consistent with the second-order resonance frequency. Rossiter 0 and Rockwell 0 posited that the upstream shear flow of the cavity was shed from the guide edge owing to the discontinuity of the geometric structure and hit the trailing edge, generating a pressure feedback phenomenon and forming the self-sustained oscillation of the shear layer. The cavity self-sustained oscillation can be divided into three types: dynamic oscillation, resonance oscillation, and elastic oscillation. The self-sustained oscillation not only causes the strong fluctuating pressure in the cavity but also produces the strong single-frequency sound. When the flow dynamic oscillation frequency coincides with a certain resonant frequency in the cavity, the coupled resonance of the standing acoustic wave is formed, and a strong "whistle" is emitted 0. Because the train model is rigid, the bogie cavity will not perform the elastic oscillation. Therefore, we can conclude that the dominant modes of the sound source for the whole car at $400 \mathrm{~Hz}$ and $800 \mathrm{~Hz}$ are probably the dynamic oscillation and the resonance oscillation, respectively. As shown from Figure 17, the sound source energy for the bottom of the head streamlined part at $800 \mathrm{~Hz}$ is obviously higher than that at $400 \mathrm{~Hz}$, which is consistent with the previous findings [43].

$$
f_{n}=\frac{n-0.25}{M+1 / 0.57} \cdot \frac{U_{0}}{L},
$$

where $f_{n}$ is the modal frequency, $U_{0}$ is the inflow velocity, $L$ is the length of the cavity along the flow direction, $M$ is Mach number, and $n$ is the modal order.

$$
f_{m}=\frac{(2 m-1) C}{4 H\left[1+0.65(L / H)^{0.75}\right]},
$$

where $f_{m}$ is the natural frequency, $C$ is the ambient sound speed, $H$ is the depth of the cavity, and $m$ is the order of the resonance frequency.

To summarize, the distribution of aerodynamic noise sources on the high-speed trains in tunnels is closely related to the characteristics of the flow field structure. In general, if the spatial distribution of velocity or the vorticity amplitude in a certain area of a high-speed train is more nonuniform and the strength of the shed vortex is stronger, the sound source intensity is stronger in that area. The dynamic oscillation and the resonance oscillation, respectively, dominate probably the mechanism of the sound source energy for the train's downside at $400 \mathrm{~Hz}$ and $800 \mathrm{~Hz}$.

\section{Conclusions}

Herein, a LES and acoustic nonreflection boundary conditions were used to simulate the fluctuating flow field around the high-speed train in an infinite tunnel. The sound source energy was calculated using the time gradient of the fluctuating pressure on the surface of the train, and the characteristics of the sound source were discussed. Further, using the sound theory of cavity flow and the flow field structure, the sound source's distribution law and its formation mechanism for the high-speed train in the tunnel are obtained. The details are as follows:

(1) The sound source energy of the upstream carriage's upside is larger than that of the downstream carriage's upside, but the opposite is true for the carriage's downside. The sound source energy of bogie 01 is far larger than that of the other bogies.

(2) The sound source energies of the whole car's downside/upside, the head car, and the six bogies account for $44.4 \%, 55.6 \%, 42.6 \%$, and $26.2 \%$ of the whole car's sound source energy, respectively.

(3) The ratio of the sound energy is approximately $1: 3.57$ between the upside and downside of the head car, 1.11:1 between the upside and downside of the middle car, and 2.38:1 between the upside and downside of the tail car. 
(4) The sound source energies of the whole car, the bottom of the head car's streamlined part, and bogie 01 are proportional to the 5.77, 5.90, and 6.11 powers of the inflow velocity, respectively. The sound source energy spectral pattern of the bottom of the head car's streamlined part has frequency similarity.

(5) The sound energy spectrum of the whole car presents broadband and multipeak characteristics. The dominant distribution frequency range is from $100 \mathrm{~Hz}$ to $4 \mathrm{kHz}$, accounting for approximately $95.1 \%$ of the total sound source energy. The peak frequencies are $400 \mathrm{~Hz}$ and $800 \mathrm{~Hz}$. The sound source energy at the peak frequency is primarily from the bogie cavity.

(6) The dynamic oscillation and the resonance oscillation, respectively, dominate probably the mechanism of the sound source energy for the train's downside at $400 \mathrm{~Hz}$ and $800 \mathrm{~Hz}$.

The external reverberation environment and interior noise of high-speed trains in tunnels can be further studied by using the acoustic source data and professional acoustic software. In addition, the comparison of the characteristics of aerodynamic noise sources for the high-speed train in tunnels and on the open track and the effect of scaling on the aerodynamic noise of high-speed trains is also of interest to everyone. These parts will be discussed in detail in the next papers.

\section{Data Availability}

The data used to support the findings of this study are available from the corresponding author upon request.

\section{Disclosure}

Tan Xiao-Ming is the first author.

\section{Conflicts of Interest}

The authors declare that they have no conflicts of interest.

\section{Acknowledgments}

This work was supported by the Ministry of Education, School of Traffic \& Transportation Engineering (2016YFB1200503-03) and (2017YFB1201103-02). The fund project of this study is Study on the Control Technology of the Comprehensive Comfort Based on the Active Noise Control (2016YFB1200503-03).

\section{References}

[1] T. D’Alfonso, C. Jiang, and V. Bracaglia, “Air transport and high-speed rail competition: environmental implications and mitigation strategies," Transportation Research Part A: Policy and Practice, vol. 92, pp. 261-276, 2015.
[2] B. D. Chiara, D. De Franco, N. Coviello, and D. Pastrone, "Comparative specific energy consumption between air transport and high-speed rail transport: a practical assessment," Transportation Research Part D: Transport and Environment, vol. 52, pp. 227-243, 2017.

[3] V. Krylov, Noise and Vibration from High-Speed Trains, Thomas Telford, London, 2001.

[4] B. Mauclaire, "Noise generated by high speed trains," in International Conference on Noise Control, pp. 371-374, Gothenburg, 1990.

[5] D. Thompson, Railway Noise and Vibration: Mechanisms, Modelling and Means of Control, Elsevier, 2009.

[6] J. Block, UK Aerodynamic Noise Measurements and Analysis, IMA6TR-051223-AEATUK (the IMAGINE Technical Report).

[7] X. Zhang, "Empirical modeling of railway aerodynamic noise using one microphone pass-by recording," in Noise and Vibration Mitigation for Rail Transportation Systems, J. Nielsen, Ed., vol. 126 of Notes on Numerical Fluid Mechanics and Multidisciplinary Design, pp. 125-131, Springer, Berlin, Heidelberg, 2015.

[8] C. Mellet, F. Létourneaux, F. Poisson, and C. Talotte, "High speed train noise emission: latest investigation of the aerodynamic/rolling noise contribution," Journal of Sound and Vibration, vol. 293, no. 3-5, pp. 535-546, 2006.

[9] B. He, X. B. Xiao, Q. Zhou, Z.-h. Li, and X.-s. Jin, "Investigation into external noise of a high-speed train at different speeds," Journal of Zhejiang University SCIENCE A, vol. 15, no. 12, pp. 1019-1033, 2014.

[10] Y. Deng, X. Xiao, B. He, and X. S. Jin, "Analysis of external noise spectrum of high-speed railway," Journal of Central South University, vol. 21, no. 12, pp. 4753-4761, 2014.

[11] N. Frémion, N. Vincent, M. Jacob, G. Robert, A. Louisot, and S. Guerrand, "Aerodynamic noise radiated by the intercoach spacing and the bogie of a high-speed train," Journal of Sound and Vibration, vol. 231, no. 3, pp. 577-593, 2000.

[12] A. Lauterbach, K. Ehrenfried, S. Loose, and C. Wagner, "Microphone array wind tunnel measurements of Reynolds number effects in high-speed train aeroacoustics," International Journal of Aeroacoustics, vol. 11, no. 3-4, pp. 411-446, 2012.

[13] S. A. Lee, H. M. Kang, Y. B. Lee, C. W. Kim, and K. H. Kim, "The aero-acoustic analysis for each part of double arm pantograph of high speed train," Journal of Computational Fluids Engineering, vol. 20, no. 2, pp. 61-66, 2015, In Korean.

[14] E. L. Iglesias, D. J. Thompson, M. Smith, T. Kitagawa, and N. Yamazaki, "Anechoic wind tunnel tests on high-speed train bogie aerodynamic noise," International Journal of Rail Transportation, vol. 5, no. 2, pp. 87-109, 2017.

[15] N. Yamazaki and A. Ido, "Evaluation methods for aerodynamic noise from a high-speed train bogie in a wind tunnel test," in INTER-NOISE and NOISE-CON Congress and Conference Proceedings, pp. 1543-1553, Osaka, Japan, 2011.

[16] M. J. Lighthill, "On sound generated aerodynamically I. General theory," Proceedings of the Royal Society A: Mathematical, Physical and Engineering Sciences, vol. 211, no. 1107 , pp. 564-587, 1952.

[17] H. H. Yu, J. C. Li, and H. Q. Zhang, "On aerodynamic noises radiated by the pantograph system of high-speed trains," Acta Mechanica Sinica, vol. 29, no. 3, pp. 399-410, 2013.

[18] J. Y. Zhu and Z. W. Hu, "The flow and flow-induced noise behavior of a simplified high-speed train leading car," in China 
Aeroacoustics Academic Conference, pp. 92-99, Beijing, China, 2016.

[19] Y. Zhang, J. Zhang, T. Li, L. Zhang, and W. Zhang, "Research on aerodynamic noise reduction for high-speed trains," Shock and Vibration, vol. 2016, Article ID 6031893, 21 pages, 2016.

[20] J. Zhang, J. Li, H. Tian, G. J. Gao, and J. Sheridan, "Impact of ground and wheel boundary conditions on numerical simulation of the high-speed train aerodynamic performance," Journal of Fluids and Structures, vol. 61, pp. 249-261, 2016.

[21] Z. Sun, J. Song, and Y. An, "Numerical simulation of aerodynamic noise generated by high speed trains," Acta Scientiarum Naturalium Universitatis Pekinensis, vol. 6, no. 2, pp. 173185, 2012.

[22] Y. Shuanbao, G. Dilong, S. Zhenxu, Y. Guowei, and C. Dawei, "Optimization design for aerodynamic elements of high speed trains," Computers \& Fluids, vol. 95, pp. 56-73, 2014.

[23] D. J. Maull and L. F. East, "Three-dimensional flow in cavities," Journal of Fluid Mechanics, vol. 16, no. 4, pp. 620-632, 1963.

[24] D. Rockwell and E. Naudascher, "Review-self-sustaining oscillations of flow past cavities," Journal of Fluids Engineering, vol. 100, no. 2, pp. 152-165, 1978.

[25] M. Ikeda and T. Mitsumoji, "Numerical estimation of aerodynamic interference between panhead and articulated frame," Quarterly Report of RTRI, vol. 50, no. 4, pp. 227-232, 2009.

[26] S. Lei, Z. Chengchun, W. Jing, and R. Luquan, "Numerical analysis of aerodynamic noise of a high-speed pantograph," in 2013 Fourth International Conference on Digital Manufacturing \& Automation, pp. 837-841, Qingdao, China, June 2013.

[27] J. Y. Zhu, Z. W. Hu, and D. J. Thompson, "The flow and flowinduced noise behavior of a simplified high-speed train bogie in the cavity with and without a fairing," Proceedings of the Institution of Mechanical Engineers, Part F: Journal of Rail and Rapid Transit, vol. 232, no. 3, pp. 759-773, 2017.

[28] E. Masson, N. Paradot, and E. Allain, "The numerical prediction of the aerodynamic noise of the TGV POS high-speed train power car," in Noise and Vibration Mitigation for Rail Transportation Systems, T. Maeda, Ed., vol. 118 of Notes on Numerical Fluid Mechanics and Multidisciplinary Design, pp. 437-444, Springer, Tokyo, 2012.

[29] D. Uystepruyst, M. William-Louis, E. Creusé, S. Nicaise, and F. Monnoyer, "Efficient 3D numerical prediction of the pressure wave generated by high-speed trains entering tunnels," Computers \& Fluids, vol. 47, no. 1, pp. 165-177, 2011.

[30] J. Niu, D. Zhou, X. Liang, T. Liu, and S. Liu, "Numerical study on the aerodynamic pressure of a metro train running between two adjacent platforms," Tunnelling and Underground Space Technology, vol. 65, pp. 187-199, 2017.

[31] Z. Nan, W. Xing, X. Hua, and L. Ya, "Research on numerical simulation approach for flow induced noise and the influence of the acoustic integral surface," Journal of Ship Mechanics, vol. 20, no. 7, pp. 892-908, 2016.

[32] A. Favre, "Equations des gaz turbulents compressibles, II. Méthode des vitesses moyennes: méthode des vitesses macroscopiques pondérées par la masse volumique," Journal de mecanique, vol. 4, no. 4, pp. 391-400, 1965.

[33] L. D. Mare and W. P. Jones, "LES of turbulent flow past a swept fence," International Journal of Heat and Fluid Flow, vol. 24, no. 4, pp. 606-615, 2003.
[34] O. V. Vasilyev, T. S. Lund, and P. Moin, "A general class of commutative filters for LES in complex geometries," Journal of Computational Physics, vol. 146, no. 1, pp. 82-104, 2001.

[35] G. W. Hedstrom, "Nonreflecting boundary conditions for nonlinear hyperbolic systems," Journal of Computational Physics, vol. 30, no. 2, pp. 222-237, 1979.

[36] K. W. Thompson, "Time dependent boundary conditions for hyperbolic systems," Journal of Computational Physics, vol. 68, no. 1, pp. 1-24, 1987.

[37] T. C. Vanajakshi, K. W. Thompson, and D. C. Black, "Boundary value problems in magnetohydrodynamics (and fluid dynamics). I. Radiation boundary condition," Journal of Computational Physics, vol. 84, no. 2, pp. 343-359, 1989.

[38] M. J. Lighthill, "On sound generated aerodynamically II. Turbulence as a source of sound," Proceedings of the Royal Society A: Mathematical, Physical and Engineering Sciences, vol. 222, no. 1148, pp. 1-32, 1148.

[39] N. Curle, "The influence of solid boundaries upon aerodynamic sound," Proceedings of the Royal Society A: Mathematical, Physical and Engineering Sciences, vol. 231, no. 1187, pp. 505-514, 1955.

[40] U. Piomelli and J. R. Chasnov, "Large-eddy simulations: theory and applications," in Turbulence and Transition Modelling, M. Hallbäck, D. S. Henningson, A. V. Johansson, and P. H. Alfredsson, Eds., vol. 2 of ERCOFTAC Series, pp. 269-336, Springer, Dordrecht, 1996.

[41] H. Nan-song, "Wind tunnel test technology for aerodynamic noise of high speed train," in China Aeroacoustics Academic Conference, pp. 9-17, Beijing, China, 2016.

[42] J. C. R. Hunt, A. A. Wray, and P. Moin, "Eddies, streams, and convergence zones in turbulent flows studying turbulence using numerical simulation databases," in Studying Turbulence Using Numerical Simulation Databases, vol. 2, pp. 193-208, Stanford, CA, USA, 1988.

[43] J. E. Rossiter, "Wind-tunnel experiments on the flow over rectangular cavities at subsonic and transonic speeds," $R$ \& $M$, vol. 3438, 1966. 


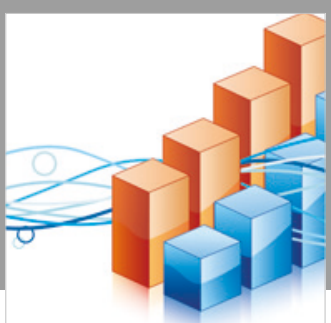

Advances in

Operations Research

\section{-n-m}
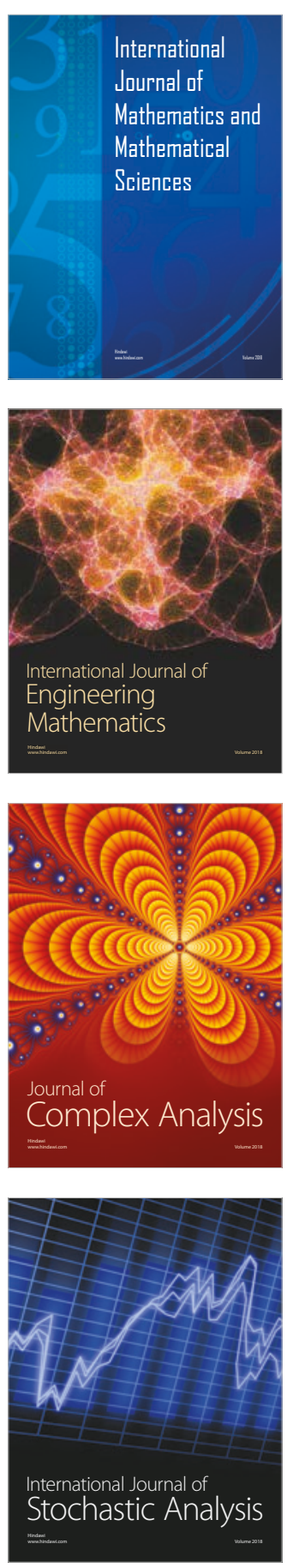
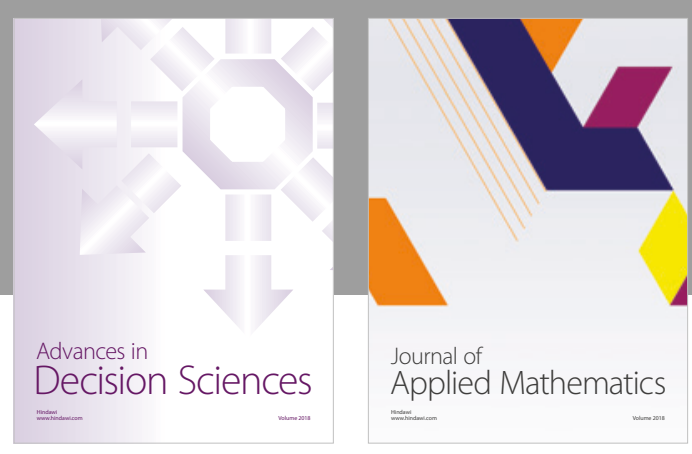

Journal of

Applied Mathematics
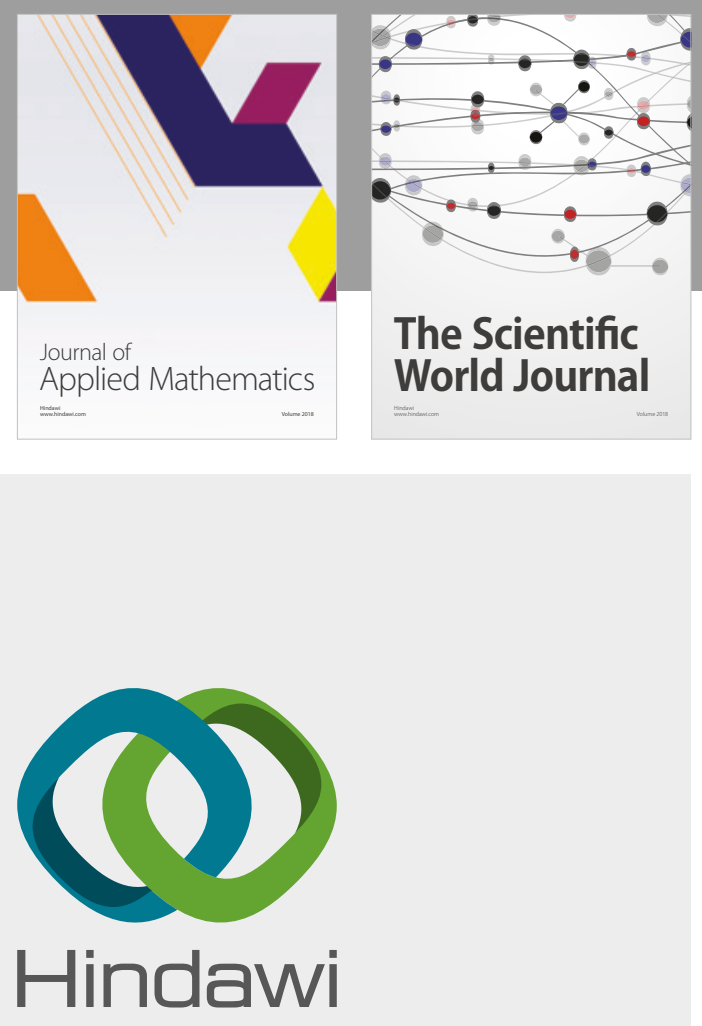

Submit your manuscripts at

www.hindawi.com

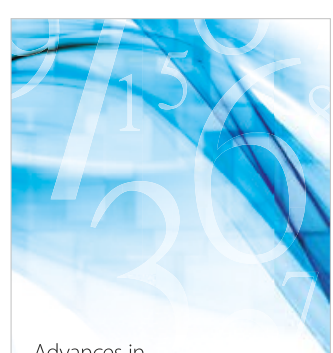

Advances in
Numerical Analysis
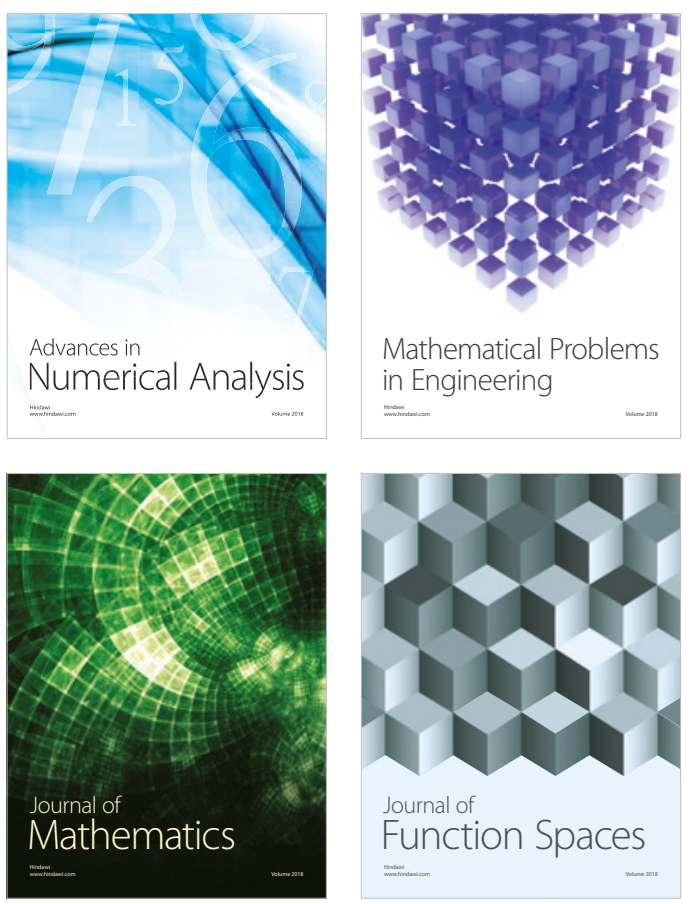

Mathematical Problems in Engineering

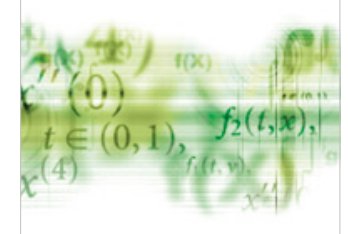

International Journal of

Differential Equations

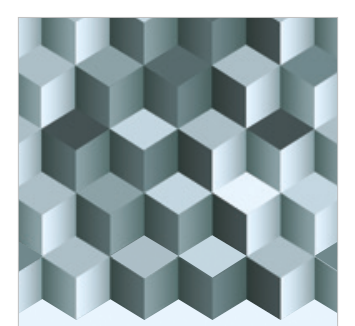

Journal of

Function Spaces
The Scientific

World Journal

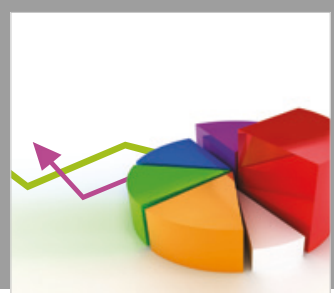

Journal of

Probability and Statistics
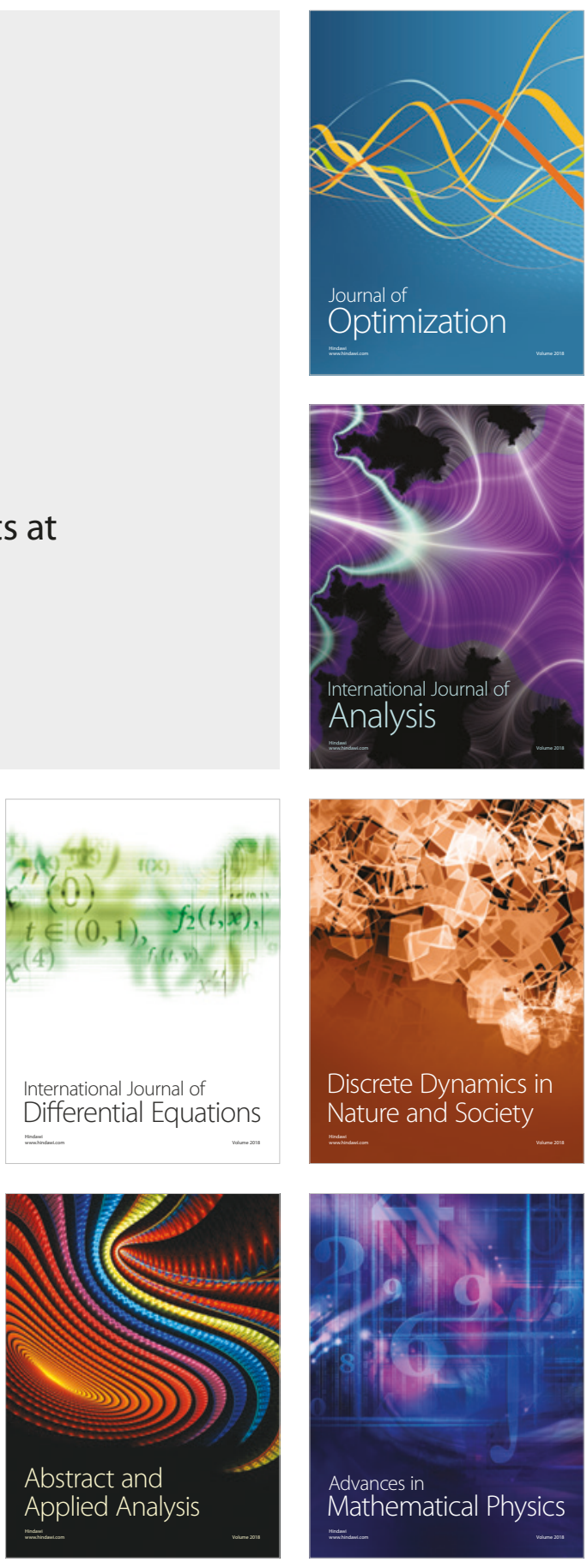